\title{
Soft collinear effective theory for heavy WIMP annihilation
}

\author{
Martin Bauer, ${ }^{a, b}$ Timothy Cohen, ${ }^{c, d, e, f}$ Richard J. Hill ${ }^{a}$ and Mikhail P. Solon ${ }^{a, g}$ \\ ${ }^{a}$ Enrico Fermi Institute and Department of Physics, \\ The University of Chicago, Chicago, Illinois, 60637, U.S.A. \\ ${ }^{b}$ Theoretical Physics Department, \\ Fermi National Accelerator Laboratory, Batavia, IL 60510, U.S.A. \\ ${ }^{c}$ Department of Physics, Princeton University, \\ Princeton, NJ, 08544, U.S.A. \\ ${ }^{d}$ School of Natural Sciences, Institute for Advanced Study, \\ Princeton, NJ, 08540, U.S.A. \\ ${ }^{e}$ Institute of Theoretical Science, University of Oregon, \\ Eugene, OR, 97403, U.S.A. \\ ${ }^{f}$ SLAC National Accelerator Laboratory, \\ Menlo Park, CA, 94025, U.S.A. \\ ${ }^{g}$ Berkeley Center for Theoretical Physics, Department of Physics, \\ and Theoretical Physics Group, Lawrence Berkeley National Laboratory, \\ University of California, Berkeley, CA 94270, U.S.A. \\ E-mail: m.bauer@thphys.uni-heidelberg.de, tcohen@uoregon.edu, \\ richardhill@uchicago.edu, mpsolon@berkeley.edu
}

ABstract: In a large class of models for Weakly Interacting Massive Particles (WIMPs), the WIMP mass $M$ lies far above the weak scale $m_{W}$. This work identifies universal Sudakov-type logarithms $\sim \alpha \log ^{2}\left(2 \mathrm{M} / \mathrm{m}_{W}\right)$ that spoil the naive convergence of perturbation theory for annihilation processes. An effective field theory (EFT) framework is presented, allowing the systematic resummation of these logarithms. Another impact of the large separation of scales is that a long-distance wavefunction distortion from electroweak boson exchange leads to observable modifications of the cross section. Careful accounting of momentum regions in the EFT allows the rigorous disentanglement of this so-called Sommerfeld enhancement from the short-distance hard annihilation process. The WIMP is described as a heavy-particle field, while the electroweak gauge bosons are treated as soft and collinear fields. Hard matching coefficients are computed at renormalization scale $\mu \sim 2 M$, then evolved down to $\mu \sim m_{W}$, where electroweak symmetry breaking is incorporated and the matching onto the relevant quantum mechanical Hamiltonian is performed. The example of an $\mathrm{SU}(2)_{W}$ triplet scalar dark matter candidate annihilating 
to line photons is used for concreteness, allowing the numerical exploration of the impact of next-to-leading order corrections and $\log$ resummation. For $M \simeq 3 \mathrm{TeV}$, the resummed Sommerfeld enhanced cross section is reduced by a factor of $\sim 3$ with respect to the treelevel fixed order result.

Keywords: Beyond Standard Model, Cosmology of Theories beyond the SM, Resummation, Effective field theories

ARXIV EPRINT: 1409.7392 


\section{Contents}

1 Introduction 1

2 Scalar model $\quad 4$

3 Fixed order matching onto quantum mechanics 5

3.1 Determining $V \quad 6$

3.2 The Sommerfeld enhancement 6

3.3 Determining $W$ : full theory $\quad 7$

$\begin{array}{lll}3.4 & \text { Determining } W \text { : quantum mechanics } & 11\end{array}$

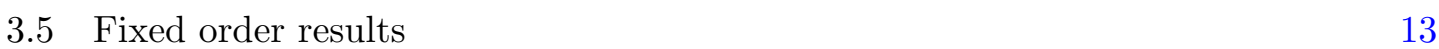

4 Deriving the effective theory $\quad 14$

$\begin{array}{lll}4.1 & \text { Regions analysis } & 14\end{array}$

4.2 Heavy particle and soft collinear effective theory for WIMP annihilation 17

$\begin{array}{ll}4.3 \text { Electroweak symmetric SCET Feynman rules } & 19\end{array}$

5 High scale matching $\quad 21$

5.1 Matching conditions 21

$\begin{array}{lll}5.2 & \text { Renormalized matching coefficients } & 23\end{array}$

6 Renormalization group evolution $\quad 25$

6.1 Anomalous dimensions 26

$\begin{array}{ll}6.2 \text { Sudakov resummation } & 27\end{array}$

7 Weak scale matching $\quad 28$

$\begin{array}{lll}7.1 & \text { Electroweak broken SCET Feynman rules } & 28\end{array}$

$\begin{array}{lll}7.2 & \text { WIMP annihilation in electroweak broken SCET } & 30\end{array}$

$\begin{array}{lll}7.3 & \text { Collinear anomaly } & 32\end{array}$

$\begin{array}{lll}7.4 & \text { Weak scale matching results } & 32\end{array}$

8 Implications $\quad 33$

9 Summary 35

\section{Introduction}

Determining the particle nature of dark matter is one of the primary goals of the particle physics community [1]. One framework that has received tremendous attention stems from the simple assumption that the dark matter communicates with the Standard Model via the weak interactions. If the Universe had a simple thermal expansion history from 
temperatures of $\gtrsim \mathrm{TeV}$ until today, ${ }^{1}$ it is natural for a Weakly Interacting Massive Particle (WIMP) to freeze out with the measured dark matter abundance (for a review, see [7]). Another attractive feature of WIMP models is that they lead to observable signatures in some combination of direct detection, indirect detection, and collider experiments.

The most studied WIMPs tend to have masses in the $\mathcal{O}(100 \mathrm{GeV})$ range. Avoiding phenomenological constraints while yielding the measured abundance often requires multi-state systems that include mass mixing [8, 9], e.g. the "well-tempered neutralino" of the Minimal Supersymmetric Standard Model (MSSM) [10]. Another compelling class of WIMP candidates consists of dark matter composed of (mostly) pure gauge eigenstates of the weak interactions. This scenario can arise from models that extend the Standard Model by only minimal field content [11-14]. If these WIMPs are thermal relics, then a hierarchy between the weak scale $m_{W}$ and the mass scale of these new particles $M$ is predicted $[11,15,16]$. Additionally, the MSSM can reproduce features of this minimal dark matter paradigm when the lightest superpartner is the pure wino or the pure Higgsino. Similar candidates can emerge from underlying composite structure [17-20].

The multi-TeV mass regime also becomes increasingly motivated as bounds from collider experiments become more stringent (e.g. for an overview in the context of supersymmetry searches at LHC8, see [21]). One interpretation of these null results is that the new physics scale will emerge somewhat higher than the weak scale. Clearly, WIMP models with $M \gg m_{W}$ deserve careful study.

From a field-theoretic point of view, this regime becomes interesting because physical processes can exhibit generic behavior as an expansion in the small ratio of scales, in the same manner that hydrogen-like atomic spectroscopy or heavy meson phenomenology exhibit universal leading order behavior in $\left(\alpha m_{e}\right) / m_{\text {nucleus }}$ or $\Lambda_{\mathrm{QCD}} / m_{\text {heavy quark }}$, respectively. This universality also emerges for heavy WIMP processes.

Heavy WIMPs are difficult to probe experimentally. Searches can be performed at the LHC, but the current mass reach is only on the order of a few hundred GeV [22-27]. Recently, it has been shown that a future collider with $\sqrt{s} \sim 100 \mathrm{TeV}$ could have some impact on the parameter space of these models, although it does not appear possible to probe masses that correspond to thermal relics [28, 29]. Direct detection prospects for heavy electroweak dark matter are also challenging. A nonvanishing cross section only appears at loop level [30-33]. Additionally, the larger mass implies a smaller number density. To make matters worse, a universal amplitude-level cancellation occurs in the heavy WIMP limit [31]. The resulting $\sim 10^{-47} \mathrm{~cm}^{2}$ cross section remains a target for next-generation direct detection searches, but these experiments will have to contend with the presence of neutrino background [34].

Fortunately, indirect detection is a viable probe of multi-TeV dark matter. In particular, photon lines that result from WIMP annihilation can be searched for using gamma ray telescopes. In part, this rate is observable due to a non-perturbative Sommerfeld

\footnotetext{
${ }^{1}$ It is plausible that the history of the Universe was more complicated in such a way that the relic density of dark matter would be impacted [2-6]. This motivates providing results for a full range of masses as opposed to restricting to the "thermal" value. Additionally, it is possible that the WIMP is a subdominant component of the dark matter.
} 
enhancement to the cross section when $\alpha_{2} M \gtrsim m_{W}[12,35-39]$, where $\alpha_{2}$ is the electroweak fine structure constant. Investigation of constraints from current experiments such as H.E.S.S. [40] indicate that under certain assumptions on the galactic dark matter halo model, some heavy WIMPs are already severely constrained from annihilation to line photons [41-43]. These conclusions depend both on the halo model and on the precise determination of the low-velocity WIMP annihilation cross section. While the former remains a subject of astrophysical study, the latter lies firmly in the domain of particle physics.

The study of heavy WIMP annihilation presents a multi-scale field theory problem, involving large corrections $\sim \alpha_{2} \log ^{2}\left(2 \mathrm{M} / \mathrm{m}_{W}\right)$ in the perturbative expansion. A complete scale separation is necessary both to obtain reliable numerical predictions for the cross section and to identify the universal features of heavy WIMP annihilation. In particular, it will be demonstrated that the dominant effect of perturbative corrections is the reduction of the tree-level amplitude by a universal factor. The dominant contribution to this universal factor can be traced to the so-called cusp anomalous dimension [44-47], which governs the renormalization of Wilson loops in gauge theory.

The annihilation amplitudes can be analyzed in an Effective Field Theory (EFT) at the operator level. Schematically, the leading operators take the form

$$
O_{\mathrm{ann}} \sim \phi_{v} \phi_{v} \mathcal{A}_{n} \mathcal{A}_{\bar{n}}
$$

where $\phi_{v}$ and $\mathcal{A}_{n}$ are EFT fields that describe the initial state non-relativistic WIMPs and the final state energetic collinear electroweak gauge bosons, respectively ( $v$ and $n, \bar{n}$ are associated timelike and lightlike vectors; detailed expressions are given in (4.19) below). Four separate field theories are necessary to capture the relevant physics, as sketched in figure 1. At renormalization scale $\mu \gg 2 M$, the full relativistic Standard Model with the addition of the WIMP sector is appropriate. Below this scale, the dynamics of the heavy WIMPs is captured by matching onto Heavy Particle EFT [48-51]. Since momentum modes with $p^{2} \gtrsim M^{2}$ are no longer present in the theory, final state particles must be restricted to have virtuality small compared to this scale. As will be demonstrated by isolating various regions of one-loop diagrams, the language of Soft Collinear Effective Theory (SCET) [52-59] captures the relevant IR dynamics of the effectively massless Standard Model fields. Given that $\mu \gg m_{W}$, it is appropriate to treat the theory in the electroweak symmetric phase, which simplifies calculations. Next, the Renormalization Group Equation (RGE) is solved, yielding the Wilson coefficients at the scale $\mu \sim m_{W}$. At this scale, electroweak symmetry breaking is relevant, and the appropriate finite corrections are computed using SCET in a field basis with broken electroweak symmetry. This procedure systematically resums large logarithms, providing a controlled perturbative expansion. The final matching step determines the parameters of a quantum mechanical Hamiltonian in which phenomenological observables may be straightforwardly computed. The EFT approach allows a rigorous definition of, and separation between, the long-distance physics associated with wavefunction distortions, i.e., the Sommerfeld enhancement, and the short-distance physics of the annihilation process. Subleading perturbative, power, and velocity corrections may be systematically incorporated. 


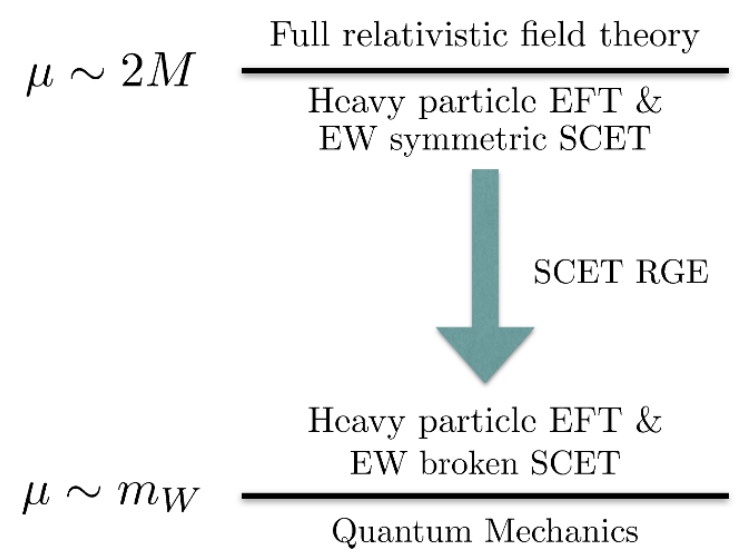

Figure 1. A schematic of the EFT decomposition utilized in this calculation.

In this paper, we focus for simplicity on the case of heavy scalar triplet WIMP annihilation to photons. The scalar triplet can be taken as a viable dark matter candidate on its own, or seen as a scalar proxy for the fermionic "wino". The wino appears as the lightest superpartner in models that involve anomaly mediated supersymmetry breaking [60, 61], and is often the dark matter candidate in models of Split Supersymmetry [62-70]. The analysis can be readily extended to describe heavy WIMPs of other spins and other electroweak quantum numbers, to describe different final states, and to compute thermal relic abundances in addition to present-day indirect detection signatures. Details involving the phenomenologically interesting case of wino annihilation to line photons will be presented in future work [71].

The remainder of the paper is structured as follows. In section 2 , we specify the scalar model. In section 3, we provide the low-energy quantum mechanical Hamiltonian and compute matching conditions in terms of free-particle annihilation amplitudes through one-loop order. This will reveal large logarithms in the matching coefficients that will be later resummed via Renormalization Group ( $R G$ ) evolution. In section 4, we perform a regions analysis of prototypical diagrams appearing in the perturbative evaluation of heavy WIMP annihilation, and introduce the relevant formalism of SCET. Section 5 gives hard matching conditions for a heavy scalar WIMP. Section 6 derives anomalous dimensions and renormalization group evolution equations governing the intermediate theory at scales $m_{W} \ll \mu \ll M$. Section 7 computes matching conditions onto the low-scale quantum mechanical theory. Section 8 gives the results for resummed physical annihilation cross sections including the Sommerfeld enhancement and investigates the impact of resummation. Section 9 provides a summary and outlook.

\section{Scalar model}

The goal of this paper is to construct and apply an EFT appropriate for heavy WIMP annihilation. While the formalism is general, for concreteness, we will consider a scalar electroweak triplet with zero hypercharge. Consider the Lagrangian for such a heavy 
scalar triplet,

$$
\mathcal{L}=\frac{1}{2}\left(D_{\mu} \phi\right)^{2}-\frac{1}{2} M^{2} \phi^{2}
$$

The covariant derivative is

$$
i D_{\mu}=i \partial_{\mu}+g_{2} W_{\mu}^{a} t^{a},
$$

where $\left(t^{a}\right)^{b c}=i \epsilon^{b a c}$ are $\mathrm{SU}(2)_{W}$ generators in the adjoint representation. In the basis of electric charge eigenstates we have

$$
i D_{\mu}=i \partial_{\mu}+e Q A_{\mu}+\frac{g_{2}}{c_{W}}\left(t^{3}-s_{W}^{2} Q\right) Z_{\mu}+\frac{g_{2}}{\sqrt{2}}\left(t^{+} W_{\mu}^{+}+t^{-} W_{\mu}^{-}\right)
$$

where $Q \equiv t^{3}+Y$ is the electric charge in units of the proton charge, and $t_{ \pm}=t_{1} \pm i t_{2}$. The Lagrangian in this basis becomes

$$
\begin{aligned}
\mathcal{L}= & \frac{1}{2}\left(\partial_{\mu} \phi_{0}\right)^{2}-\frac{1}{2} M^{2} \phi_{0}^{2}+\partial_{\mu} \phi_{+} \partial^{\mu} \phi_{-}-M^{2} \phi_{+} \phi_{-}-i g_{2} W_{\mu}^{+}\left(\phi_{-} \partial^{\mu} \phi_{0}-\phi_{0} \partial^{\mu} \phi_{-}\right) \\
& -i g_{2} W_{\mu}^{-}\left(-\phi_{+} \partial^{\mu} \phi_{0}+\phi_{0} \partial^{\mu} \phi_{+}\right)+i e A_{\mu}\left(\phi_{-} \partial^{\mu} \phi_{+}-\phi_{+} \partial^{\mu} \phi_{-}\right) \\
& +i g_{2} c_{W} Z_{\mu}\left(\phi_{-} \partial^{\mu} \phi_{+}-\phi_{+} \partial^{\mu} \phi_{-}\right)+\phi_{+} \phi_{-}\left(e^{2} A_{\mu} A^{\mu}+2 e g_{2} c_{W} A_{\mu} Z^{\mu}+g_{2}^{2} c_{W}^{2} Z_{\mu} Z^{\mu}\right) \\
& -\phi_{0}\left(\phi_{-} W_{\mu}^{+}+\phi_{+} W_{\mu}^{-}\right)\left(e g_{2} A^{\mu}+g_{2}^{2} c_{W} Z^{\mu}\right)-\frac{1}{2} g_{2}^{2}\left[\left(\phi_{-}\right)^{2} W_{\mu}^{+} W^{+\mu}+\left(\phi_{+}\right)^{2} W_{\mu}^{-} W^{-\mu}\right] \\
& +g_{2}^{2}\left(\phi_{0}^{2}+\phi_{+} \phi_{-}\right) W_{\mu}^{+} W^{-\mu},
\end{aligned}
$$

from which it is straightforward to read off the Feynman rules. We neglect renormalizable self-couplings of the scalar field, $\sim \phi^{4}$, and Higgs interactions, $\sim H^{\dagger} H \phi^{2}$. It would be straightforward to include these couplings in an extended analysis.

\section{Fixed order matching onto quantum mechanics}

To begin, let us match the WIMP annihilation process computed directly in the high scale field theory onto a quantum mechanical Hamiltonian. This will make clear the separation between the hard annihilation process and the wavefunction distortion. The former arises from offshell momentum regions of loop diagrams, and is represented by contributions to contact interactions in the quantum mechanical Hamiltonian. The latter emerges from nearly onshell momentum regions, and is reproduced by corresponding quantum mechanical potentials.

The general quantum mechanical Hamiltonian appropriate for the center-of-mass frame for the two-particle system takes the form ${ }^{2}$

$$
H=\frac{p^{2}}{2 M_{r}}+\Delta+V+i W
$$

where $V$ and $W$ are Hermitian, $M_{r}$ denotes the reduced mass, and $\Delta$ is the residual mass matrix, which captures the difference in rest mass energy between the states of interest. In

\footnotetext{
${ }^{2}$ See e.g. [72]. A related formalism for treating velocity corrections in WIMP annihilation is given in $[73,74]$.
} 
matrix notation, acting on two components in the neutral-neutral (00) and charged-charged $(+-)$ sectors, the kinetic energy and residual mass terms are

$$
\frac{p^{2}}{2 M_{r}}+\Delta=p^{2}\left(\begin{array}{cc}
\frac{1}{M_{0}} & 0 \\
0 & \frac{1}{M_{ \pm}}
\end{array}\right)+\left(\begin{array}{cc}
0 & 0 \\
0 & 2 \delta
\end{array}\right)
$$

where the zero of energy is taken as $2 M_{0}$ and we define $\delta=M_{ \pm}-M_{0}$. For notational convenience we will set $M_{0} \equiv M$ in the following. The potential $V+i W$ is determined by comparing the Born series computed from this Hamiltonian,

$$
\left\langle\boldsymbol{k}^{\prime}|T| \boldsymbol{k}\right\rangle=\left\langle\boldsymbol{k}^{\prime}|V+i W| \boldsymbol{k}\right\rangle+\ldots,
$$

with the field theory prediction for the scattering amplitude.

\subsection{Determining $V$}

The Hermitian potential $V$ will capture the effects of the long range force experienced by the WIMPs, and $W$ will encode the hard annihilation process via the optical theorem as discussed in section 3.3. Employing the Feynman rules for heavy scalars from (2.4), the result for $V$ reads

$$
\left\langle\boldsymbol{k}^{\prime}|V| \boldsymbol{k}\right\rangle=\left(\begin{array}{cc}
0 & -4 \pi \alpha_{2}\left[\frac{1}{\left(\boldsymbol{k}^{\prime}-\boldsymbol{k}\right)^{2}+m_{W}^{2}}+\frac{1}{\left(\boldsymbol{k}^{\prime}+\boldsymbol{k}\right)^{2}+m_{W}^{2}}\right] \\
-4 \pi \alpha_{2}\left[\frac{1}{\left(\boldsymbol{k}^{\prime}-\boldsymbol{k}\right)^{2}+m_{W}^{2}}+\frac{1}{\left(\boldsymbol{k}^{\prime}+\boldsymbol{k}\right)^{2}+m_{W}^{2}}\right] & -4 \pi \alpha\left[\frac{1}{\left(\boldsymbol{k}^{\prime}-\boldsymbol{k}\right)^{2}+m_{\gamma}^{2}}+\frac{t_{W}^{-2}}{\left(\boldsymbol{k}^{\prime}-\boldsymbol{k}\right)^{2}+m_{Z}^{2}}\right]
\end{array}\right),
$$

where $\alpha_{2}=g_{2}^{2} / 4 \pi$ and $\alpha=e^{2} / 4 \pi$ are the electroweak and electromagnetic fine structure constants, $m_{W}$ and $m_{Z}$ are the $W^{ \pm}$and $Z^{0}$ boson masses, and $m_{\gamma}$ is an infinitesimal photon mass that is used to regulate IR divergences. In the quantum field theory calculation, the two terms in the off-diagonal elements of (3.4) arise from crossed and uncrossed diagrams involving $W^{ \pm}$exchange, and the terms in the lower right entry are from photon and $Z^{0}$ exchange, respectively. Equation (3.4) will be used in the old-fashioned perturbation theory analysis, presented in section 3.4 below, in order to determine the correct matching onto quantum mechanics at one-loop order.

\subsection{The Sommerfeld enhancement}

In order to compute the Sommerfeld enhancement, it is useful to Fourier transform $V$ from (3.4) into position space,

$$
V^{S \text {-wave }}=\left(\begin{array}{cc}
0 & -\sqrt{2} \frac{\alpha_{2}}{r} e^{-m_{W} r} \\
-\sqrt{2} \frac{\alpha_{2}}{r} e^{-m_{W} r} & -\frac{\alpha}{r}-\frac{\alpha_{2} c_{W}^{2}}{r} e^{-m_{Z} r}
\end{array}\right)
$$

where this result is appropriate for $S$-wave scattering states (at $m_{\gamma}=0$ ). Then this matrix can be used as the input to the $S$-wave Schrödinger equation to model the wavefunctions of the neutral and charged WIMP pairs, yielding the Sommerfeld enhancement. Specifically, we use the formalism outlined in the appendix of [75] to compute the physical annihilation 
cross section from quantum mechanics, using (3.1) as an input. Indices $i, j=1,2$ refer to the $(00),(+-)$ states respectively. For the wavefunction $\left(\psi^{i}\right)_{j}$, the index $i$ labels the asymptotic state and $j$ is the component index for the resulting solution. Given a choice of $i$, the boundary conditions employed are

$$
\begin{aligned}
\left(\psi^{i}(0)\right)_{j} & \rightarrow \delta_{i j}, \quad j=1,2, \\
\left(\psi^{i}(\infty)\right)_{1} & \rightarrow e^{i k_{i} r}, \\
\left(\psi^{i}(\infty)\right)_{2} & \rightarrow \begin{cases}\psi_{\text {Coulomb }}^{i}: E \geq \delta_{i} \\
e^{-\left|k_{i}\right| r} \quad: E<\delta_{i}\end{cases}
\end{aligned}
$$

where $k_{i}=M \sqrt{1-\delta_{i} / E}, E$ is the kinetic energy of the WIMP system, $\delta_{1}=0, \delta_{2}=\delta$, and $\psi_{\text {Coulomb }}^{i}$ is the wavefunction for the Coulomb scattering solution that depends on momentum $k_{i} .{ }^{3}$ Once the solutions $\psi$ have been obtained, the Sommerfeld enhancement matrix is given by

$$
s_{i j}=\left(\psi^{i}(\infty)\right)_{j}
$$

The cross section can then be computed using

$$
\left(\sigma_{i} v\right)^{\mathrm{S}-\text { wave }}=-2 \sum_{j, j^{\prime}} s_{i j} W_{j j^{\prime}}^{S \text {-wave }} s_{i j^{\prime}}^{*}
$$

where $W^{S \text {-wave }}$ denotes the absorptive part of the potential for $S$-wave scattering states. ${ }^{4}$

The couplings and masses are defined as their onshell values. In particular, here we are using the shorthand $\alpha_{2}=\alpha s_{W}^{-2}$ with $s_{W}^{2}=1-c_{W}^{2}$ and $c_{W}=m_{W} / m_{Z}$. All that is required to determine an annihilation cross section are (Particle Data Group [76]) inputs for $\alpha$, the $W^{ \pm}$and $Z^{0}$ masses, the WIMP mass $M$, the charged-neutral mass splitting $\delta$, the relative velocity $v$, and the $2 \times 2$ Hermitian matrix $W$. Now that the formalism for calculating the wavefunction factors has been explained, we move to the determination of the hard-annihilation contribution to the potential $W$ through one-loop order by matching field theory onto quantum mechanics.

\subsection{Determining $W$ : full theory}

The most straightforward way to determine the absorptive part of the potential, $W$, from field theory is through use of the optical theorem. Matching is done at a convenient kinematic point, specifically the two-particle threshold for neutral or charged WIMPs for diagonal elements of $W$, or at the two-particle charged WIMP threshold for off-diagonal elements (such that the amplitude describes an onshell physical process).

\footnotetext{
${ }^{3}$ Note that to achieve numerical stability, we furthermore strip off the asymptotic, plane-wave or Coulomb, factors as outlined in the appendix of [41].

${ }^{4}$ For the contact interaction $W$, this amounts to the replacements $W_{11} \rightarrow W_{11} / 2, W_{12} \rightarrow W_{12} / \sqrt{2}$, $W_{21} \rightarrow W_{21} / \sqrt{2}, W_{22} \rightarrow W_{22}$ starting from the plane wave basis (3.20).
} 


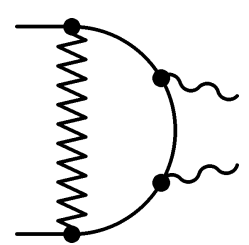

(a)

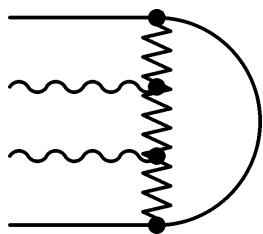

(e)

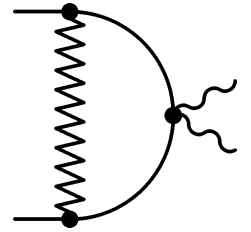

(b)

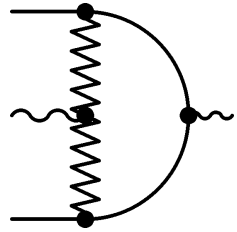

(c)

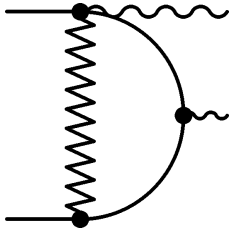

(d)

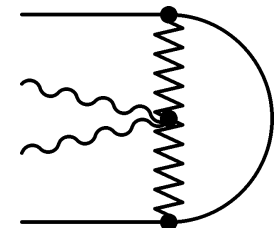

(f)

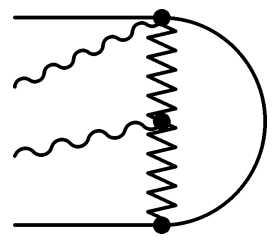

(g)

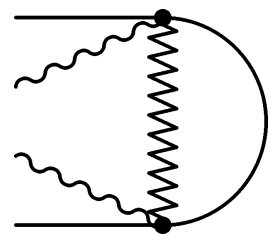

(h)

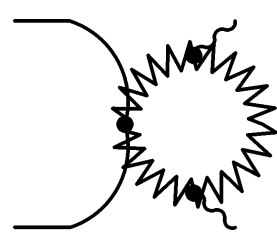

(i)

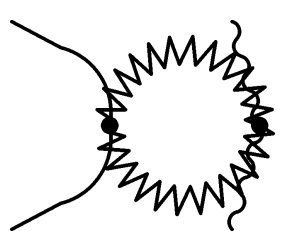

$(\mathrm{j})$

Figure 2. Diagrams contributing to hard scale matching for neutral WIMPs. Wavy lines are photons, zigzag lines are $W^{ \pm}$bosons.

The discontinuity arising from two-photon final states is found to be

$$
\begin{aligned}
i \operatorname{Disc}_{\mathrm{NR}}\left([\phi \phi]_{i} \rightarrow[\phi \phi]_{f}\right) & = \\
& =-\frac{1}{8 \pi} \frac{1}{\left(\sqrt{2 E_{i}}\right)^{2}\left(\sqrt{2 E_{f}}\right)^{2}} \mathcal{M}\left([\phi \phi]_{i} \rightarrow \gamma \gamma\right) \mathcal{M}\left([\phi \phi]_{f} \rightarrow \gamma \gamma\right)^{*}
\end{aligned}
$$

where the factors $\sqrt{2 E}$ for each external particle convert to nonrelativistic state normalization (denoted by subscript "NR"), and we have introduced the reduced amplitude, $\mathcal{M}\left([\phi \phi]_{i} \rightarrow \gamma(\epsilon) \gamma\left(\epsilon^{\prime}\right)\right)=\epsilon^{*} \cdot \epsilon^{\prime *} \mathcal{M}\left([\phi \phi]_{i} \rightarrow \gamma \gamma\right)$. Identifying Disc $\mathcal{M}=2 i$ Abs $\mathcal{M}$ gives the absorptive contribution from field theory. ${ }^{5}$

For neutral WIMP annihilation, the relevant amputated loop diagrams are shown in figure 2. Considering kinematics at both the neutral and charged WIMP thresholds, we have

$$
\begin{aligned}
\mathcal{M}^{00 \rightarrow \gamma \gamma}=\frac{e^{2} g_{2}^{2}}{(4 \pi)^{2}}\{ & C_{\text {potential }}+(16-16 i \pi) \log \frac{m_{W}}{2 M}-4-\pi^{2}+\frac{86 \pi}{3} \frac{m_{W}}{M} \\
& +\frac{m_{W}^{2}}{M^{2}}\left[26 \log ^{2} \frac{m_{W}}{2 M}+(20+10 i \pi) \log \frac{m_{W}}{2 M}-\frac{104}{3}-\frac{15 \pi^{2}}{2}-\frac{7 i \pi}{2}\right] \\
& \left.+\mathcal{O}\left(\alpha, \frac{\delta}{m_{W}}, \sqrt{\frac{\delta}{M}}, \frac{m_{W}^{3}}{M^{3}}\right)\right\},
\end{aligned}
$$

\footnotetext{
${ }^{5}$ For a single channel, the absorptive part is identified with the imaginary part, $\operatorname{Abs} \mathcal{M} \equiv \operatorname{Im} \mathcal{M}$.
} 
where $C_{\text {potential }}$ depends on whether the matrix element is evaluated at the neutral or charged WIMP threshold:

$$
C_{\text {potential }}=\left\{\begin{array}{ll}
\frac{16 \pi M}{m_{W}+\sqrt{2 M \delta}} & \text { for }\left(p+p^{\prime}\right)^{2}=4 M_{0}^{2} \\
\frac{16 \pi M}{\sqrt{2 M \delta}} \arctan \left(\frac{\sqrt{2 M \delta}}{m_{W}}\right) & \text { for }\left(p+p^{\prime}\right)^{2}=4 M_{ \pm}^{2}
\end{array} .\right.
$$

We have here ignored higher order corrections involving the mass splitting (cf. (3.17) below). For charged WIMP annihilation, the process has a tree-level contribution. Including the tree vertex with counterterms, together with the loop diagrams of figure 3 ,

$$
\begin{aligned}
&\left.\mathcal{M}^{+-\rightarrow \gamma \gamma}\right|_{\left(p+p^{\prime}\right)^{2}=4 M_{ \pm}^{2}} \\
&=Z_{2}^{\phi}\left(Z_{1}^{W}\right)^{2}\left(Z_{2}^{W}\right)^{-2} 2 e^{2}+\frac{e^{2} g_{2}^{2}}{(4 \pi)^{2}}\left\{\frac{8 \pi c_{W}^{2} M}{m_{Z}}+\frac{8 \pi s_{W}^{2} M}{m_{\gamma}}\right. \\
& \quad+8\left(c_{W}^{2} \log \frac{m_{Z}}{2 M}+s_{W}^{2} \log \frac{m_{\gamma}}{2 M}\right)-16 \log ^{2} \frac{m_{W}}{2 M}-16 \log \frac{m_{W}}{2 M}-8 i \pi \log \frac{m_{W}}{2 M} \\
&+\frac{3 \pi^{2}}{2}-18+\frac{m_{W}}{M}\left[-4 \pi+\frac{7 \pi}{3} c_{W}\right]+\frac{m_{W}^{2}}{M^{2}}\left[5 \log ^{2} \frac{m_{W}}{2 M}-12 \log \frac{m_{W}}{2 M}-2 \log \frac{m_{Z}}{2 M}\right. \\
&\left.\left.\quad+5 i \pi \log \frac{m_{W}}{2 M}-12 \log 2+\frac{20}{3}-\frac{5 \pi^{2}}{4}-\frac{7 i \pi}{4}\right]+\mathcal{O}\left(\alpha, m_{\gamma}, \frac{\delta}{m_{W}}, \sqrt{\frac{\delta}{M}}, \frac{m_{W}^{3}}{M^{3}}\right)\right\} .
\end{aligned}
$$

The renormalization constant $Z_{2}^{\phi}$ is inherited from the electroweak symmetric Lagrangian (2.1) and $Z_{1}^{W}, Z_{2}^{W}$ are field and coupling renormalization factors for the $\mathrm{SU}(2)_{W}$ gauge field [77]. ${ }^{6}$

Let us briefly review the renormalization for the scalar triplet. The 1PI two-point functions for the charged and neutral scalar fields at one-loop order are given by in dimensional regularization $(d=4-2 \epsilon)$

$$
\begin{aligned}
& -i \Sigma_{2}^{\phi_{+}}\left(p^{2}\right)=\left[c_{\epsilon}\right]\left\{\frac{2(2-\epsilon)}{\epsilon(1-\epsilon)}\left[e^{2}\left(m_{\gamma}^{2}\right)^{1-\epsilon}+g_{2}^{2} c_{W}^{2}\left(m_{Z}^{2}\right)^{1-\epsilon}+g_{2}^{2}\left(m_{W}^{2}\right)^{1-\epsilon}\right]\right. \\
& +\frac{1}{\epsilon(1-\epsilon)}\left[e^{2}\left(-2\left(m_{\gamma}^{2}\right)^{1-\epsilon}+\left(M^{2}\right)^{1-\epsilon}\right)+g_{2}^{2} c_{W}^{2}\left(-2\left(m_{Z}^{2}\right)^{1-\epsilon}+\left(M^{2}\right)^{1-\epsilon}\right)\right. \\
& \left.+g_{2}^{2}\left(-2\left(m_{W}^{2}\right)^{1-\epsilon}+\left(M^{2}\right)^{1-\epsilon}\right)\right] \\
& -\frac{1}{\epsilon}\left(M^{2}\right)^{-\epsilon}\left[e^{2}\left(2 p^{2}+2 M^{2}-m_{\gamma}^{2}\right) I\left(m_{\gamma} / M, p^{2} / M^{2}\right)\right. \\
& +g_{2}^{2} c_{W}^{2}\left(2 p^{2}+2 M^{2}-m_{Z}^{2}\right) I\left(m_{Z} / M, p^{2} / M^{2}\right) \\
& \left.\left.+g_{2}^{2}\left(2 p^{2}+2 M^{2}-m_{W}^{2}\right) I\left(m_{W} / M, p^{2} / M^{2}\right)\right]\right\}, \\
& -i \Sigma_{2}^{\phi_{0}}\left(p^{2}\right)=\left[c_{\epsilon}\right] g_{2}^{2}\left\{\frac{4(2-\epsilon)}{\epsilon(1-\epsilon)}\left(m_{W}^{2}\right)^{1-\epsilon}+\frac{2}{\epsilon(1-\epsilon)}\left[-2\left(m_{W}^{2}\right)^{1-\epsilon}+\left(M^{2}\right)^{1-\epsilon}\right]\right. \\
& \left.-\frac{2}{\epsilon}\left(M^{2}\right)^{-\epsilon}\left(2 p^{2}+2 M^{2}-m_{W}^{2}\right) I\left(m_{W} / M, p^{2} / M^{2}\right)\right\},
\end{aligned}
$$

\footnotetext{
${ }^{6}$ Following the conventions of [77], bare Lagrangian fields and parameters are given by $\left(W_{\mu}^{a}\right)^{\text {bare }}=$ $\left(Z_{2}^{W}\right)^{1 / 2} W_{\mu}^{a}, g_{2}^{\text {bare }}=Z_{1}^{W}\left(Z_{2}^{W}\right)^{-3 / 2} g_{2}$.
} 


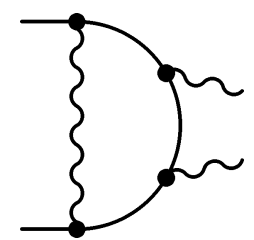

(a)

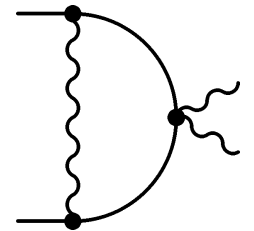

(b)

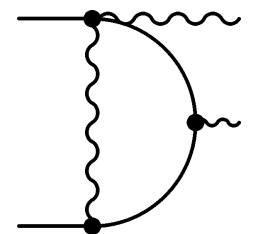

(c)

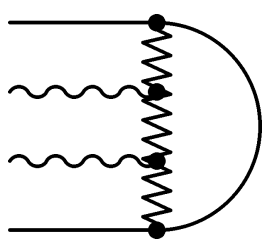

(d)

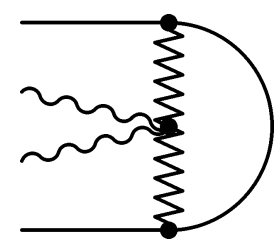

(e)

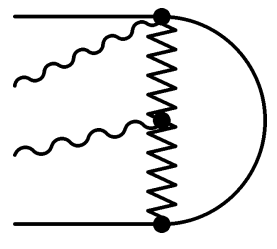

(f)

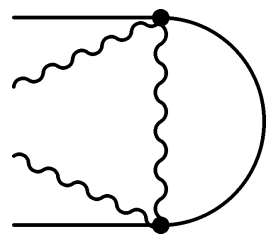

(g)

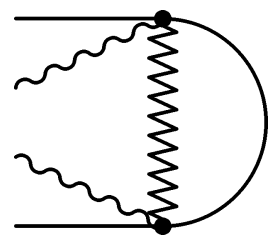

(h)

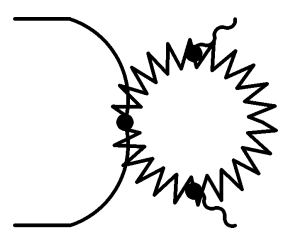

(i)

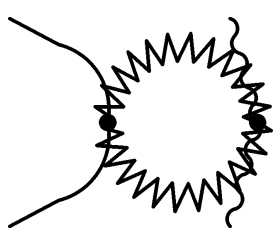

(j)

Figure 3. Diagrams contributing to matching for charged WIMPs. Wavy lines are photons, zigzag lines are $W^{ \pm}$bosons, and the inclusion of diagrams where internal photon lines are replaced by $Z^{0}$ boson lines is implied.

where we introduce the shorthand $\left[c_{\epsilon}\right]=i(4 \pi)^{-2+\epsilon} \Gamma(1+\epsilon)$, and

$$
\begin{aligned}
I\left(m, p^{2}\right) & =\int_{0}^{1} d x\left[-x(1-x) p^{2}+x+(1-x) m^{2}\right]^{-\epsilon} \\
& =1-\epsilon\left[m \sqrt{4-m^{2}} \arctan \left(\frac{\sqrt{4-m^{2}}}{m}\right)+m^{2} \log m-2\right]+\mathcal{O}\left(\epsilon^{2}\right) .
\end{aligned}
$$

From these results, it is straightforward to derive the one-loop expressions for the mass splitting,

$$
M_{ \pm}^{2}-M_{0}^{2}=\Sigma_{2}^{\phi_{+}}\left(M_{ \pm}^{2}\right)-\Sigma_{2}^{\phi_{0}}\left(M_{0}^{2}\right)=\alpha m_{W} M \frac{1-c_{W}}{s_{W}^{2}}+\mathcal{O}\left(\alpha^{2}, 1 / M^{3}\right),
$$

and the residue of the charged propagator

$$
\begin{aligned}
& Z_{2}^{\phi_{+}}-1=\left.\frac{\partial \Sigma_{2}^{\phi_{+}}}{\partial p^{2}}\right|_{p^{2}=M^{2}} \\
&=i g_{2}^{2}\left[c_{\epsilon}\right] M^{-2 \epsilon}\left[-\frac{4}{\epsilon}+4\left(s_{W}^{2} \log \frac{m_{\gamma}}{M}+c_{W}^{2} \log \frac{m_{Z}}{M}+\log \frac{m_{W}}{M}\right)\right. \\
&\left.\quad-\frac{\pi m_{W}}{M}\left(1+c_{W}\right)-\frac{3 m_{W}^{2}}{M^{2}}\left(\log \frac{m_{W}}{M}+\log \frac{m_{Z}}{M}\right)+\mathcal{O}\left(1 / M^{3}, \epsilon\right)\right] .
\end{aligned}
$$


Finally, for the combination of renormalization constants $\left(Z_{1}^{W}\right)^{2}\left(Z_{2}^{W}\right)^{-2}$ appearing in (3.12), we have

$$
2 \delta Z_{1}^{W}-2 \delta Z_{2}^{W}=-\frac{2}{s_{W} c_{W}} \frac{\Sigma^{A Z}(0)}{m_{Z}^{2}}=-\frac{g_{2}^{2}}{(4 \pi)^{2}} m_{W}^{-2 \epsilon} \frac{4}{\epsilon} .
$$

In particular, $\Sigma^{A Z}(0)$ receives contributions only from the $W^{ \pm}$boson loop, and is independent of the additional scalar triplet.

The amplitudes (3.12), (3.14) and renormalization constants (3.18), (3.19) determine the physical one-loop amplitudes for heavy scalar annihilation to photons in terms of physical parameters $\alpha, m_{W}, m_{Z}, M, \delta$. One can see from these equations that there are factors of the type $M / m_{W}$ that result from the so-called potential region of the loop integrals. It is exactly these factors that are resummed by including the Sommerfeld enhancement. Isolating the hard annihilation contribution to the $W$ matrix from terms that derive from the potential region requires working to higher order in quantum mechanics. This is the subject of the next subsection, where the equivalent quantum mechanics calculation is performed.

\subsection{Determining $W$ : quantum mechanics}

In this subsection, the matching conditions for the absorptive part of the potential $W$ are computed in quantum mechanics. Working in the plane wave basis, we write

$$
\left\langle\boldsymbol{k}^{\prime}\left|W^{(\gamma)}\right| \boldsymbol{k}\right\rangle \equiv\left(\begin{array}{cc}
w_{00}^{(\gamma)} & w_{00 ; \pm}^{(\gamma)} \\
w_{ \pm ; 00}^{(\gamma)} & w_{ \pm}^{(\gamma)}
\end{array}\right)
$$

where $w_{ \pm ; 00}=w_{00 ; \pm}^{*}$, and the superscript $(\gamma)$ denotes restriction to $\gamma \gamma$ final states. We work through lowest non-vanishing order in $\alpha$ for each of the elements $w_{i j}^{(\gamma)}$, but will also retain the first subleading term for $w_{ \pm}^{(\gamma)}$ so that our computation contains complete one-loop corrections (see (3.27) for explicit expressions). Working in the framework of "old-fashioned" perturbation theory, the nonrelativistic scattering amplitude is given by the Born series for the matrix valued potential of (3.1). What follows is the explicit computation of these matrix elements. In the following, we restrict to $\gamma \gamma$ final states and omit the superscript on $w_{i j}$.

For the charged channel:

$$
\begin{aligned}
& { }_{ \pm}\left\langle\boldsymbol{k}^{\prime}|T| \boldsymbol{k}\right\rangle_{ \pm} \\
& =i W_{ \pm}+i W_{ \pm} \otimes V_{ \pm}+V_{ \pm} \otimes i W_{ \pm}+\mathcal{O}\left(\alpha^{4}\right) \\
& ={ }_{ \pm}\left\langle\boldsymbol{k}^{\prime}|i W| \boldsymbol{k}\right\rangle_{ \pm}+\int \frac{d^{3} p}{(2 \pi)^{3}} \int \frac{d^{3} p^{\prime}}{(2 \pi)^{3}}\left\langle\boldsymbol{k}^{\prime}|V| \boldsymbol{p}\right\rangle_{ \pm \pm}\left\langle\boldsymbol{p}\left|\left(E-H_{0}\right)^{-1}\right| \boldsymbol{p}^{\prime}\right\rangle_{ \pm \pm}\left\langle\boldsymbol{p}^{\prime}|i W| \boldsymbol{k}\right\rangle_{ \pm} \\
& \quad+\int \frac{d^{3} p}{(2 \pi)^{3}} \int \frac{d^{3} p^{\prime}}{(2 \pi)^{3}}\left\langle\boldsymbol{k}^{\prime}|i W| \boldsymbol{p}\right\rangle_{ \pm \pm}\left\langle\boldsymbol{p}\left|\left(E-H_{0}\right)^{-1}\right| \boldsymbol{p}^{\prime}\right\rangle_{ \pm \pm}\left\langle\boldsymbol{p}^{\prime}|V| \boldsymbol{k}\right\rangle_{ \pm}
\end{aligned}
$$




$$
\begin{aligned}
= & i w_{ \pm}+\int \frac{d^{3} p}{(2 \pi)^{3}}\left\{i w_{ \pm}\left(\frac{\boldsymbol{k}^{2}}{M_{ \pm}}-\frac{\boldsymbol{p}^{2}}{M_{ \pm}}\right)^{-1}(-4 \pi \alpha)\left[\frac{1}{(\boldsymbol{p}-\boldsymbol{k})^{2}+m_{\gamma}^{2}}+\frac{t_{W}^{-2}}{(\boldsymbol{p}+\boldsymbol{k})^{2}+m_{Z}^{2}}\right]\right. \\
& \left.+(-4 \pi \alpha)\left[\frac{1}{\left(\boldsymbol{p}-\boldsymbol{k}^{\prime}\right)^{2}+m_{\gamma}^{2}}+\frac{t_{W}^{-2}}{\left(\boldsymbol{p}+\boldsymbol{k}^{\prime}\right)^{2}+m_{Z}^{2}}\right]\left(\frac{\boldsymbol{k}^{2}}{M_{ \pm}}-\frac{\boldsymbol{p}^{2}}{M_{ \pm}}\right)^{-1} i w_{ \pm}\right\} \cdot
\end{aligned}
$$

Here the circular blob denotes insertion of $i W$, while the elliptical blob denotes insertion of $V$. For charged particle production at threshold, $\boldsymbol{k}=\boldsymbol{k}^{\prime}=0$, this gives

$$
{ }_{ \pm}\left\langle\boldsymbol{k}^{\prime}|T| \boldsymbol{k}\right\rangle_{ \pm} \rightarrow i w_{ \pm}+2 i \alpha w_{ \pm} M_{ \pm}\left(\frac{1}{m_{\gamma}}+\frac{t_{W}^{-2}}{m_{Z}}\right)+\mathcal{O}\left(\alpha^{3}\right),
$$

where $m_{\gamma}$ is a photon mass regulating IR divergences.

For the mixed channel:

$$
\begin{aligned}
{ }_{ \pm}\left\langle\boldsymbol{k}^{\prime}|T| \boldsymbol{k}\right\rangle_{00} & \rightarrow \\
=i W_{ \pm ; 00}+i W_{ \pm} \otimes V_{ \pm ; 00}+\mathcal{O}\left(\alpha^{4}\right) & \ldots \\
=i w_{ \pm ; 00} & +\int \frac{d^{3} p}{(2 \pi)^{3}} i w_{ \pm}\left(\frac{\boldsymbol{k}^{2}}{M_{0}}-\frac{\boldsymbol{p}^{2}}{M_{ \pm}}-2 \delta\right)^{-1} \\
& \times\left(-4 \pi \alpha s_{W}^{-2}\right)\left[\frac{1}{(\boldsymbol{p}-\boldsymbol{k})^{2}+m_{W}^{2}}+\frac{1}{(\boldsymbol{p}+\boldsymbol{k})^{2}+m_{W}^{2}}\right] .
\end{aligned}
$$

Evaluated at the threshold for charged particle production, $\boldsymbol{k}^{\prime}=0$ and $\boldsymbol{k}^{2}=2 M_{0} \delta$, this expression yields

$$
{ }_{ \pm}\left\langle\boldsymbol{k}^{\prime}|T| \boldsymbol{k}\right\rangle_{00} \rightarrow i w_{ \pm ; 00}+2 i \alpha s_{W}^{-2} w_{ \pm} \frac{1}{\sqrt{2 M_{0} \delta}} \arctan \left(\frac{\sqrt{2 M_{0} \delta}}{m_{W}}\right)+\mathcal{O}\left(\alpha^{4}\right)
$$

For the neutral channel:

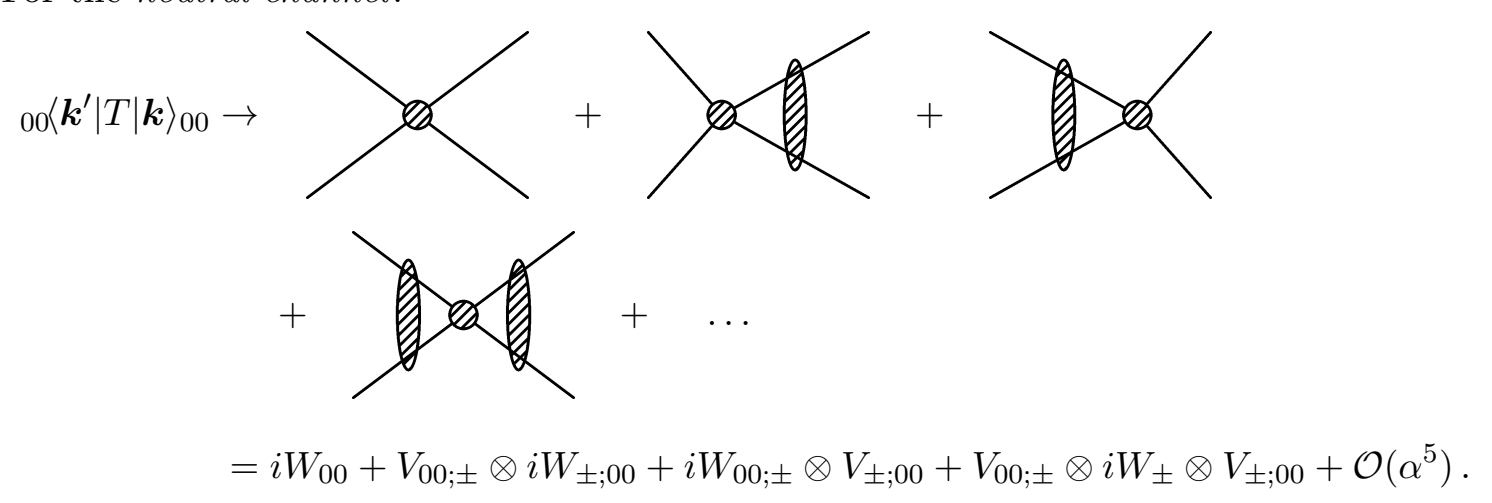

Evaluating this expression at the neutral threshold, $\boldsymbol{k}=\boldsymbol{k}^{\prime}=0$, yields

$$
\begin{aligned}
{ }_{00}\left\langle\boldsymbol{k}^{\prime}|T| \boldsymbol{k}\right\rangle_{00} \rightarrow i w_{00}+ & 4 i \alpha s_{W}^{-2} \frac{M_{ \pm}}{m_{W}+\sqrt{2 M_{ \pm} \delta}} \operatorname{Re}\left(w_{ \pm ; 00}\right) \\
+ & {\left[2 \alpha s_{W}^{-2} \frac{M_{ \pm}}{m_{W}+\sqrt{2 M_{ \pm} \delta}}\right]^{2} i w_{ \pm}+\mathcal{O}\left(\alpha^{5}\right) . }
\end{aligned}
$$


Note that $T=-\mathcal{M}_{\mathrm{NR}}$ in the conventions employed here. ${ }^{7}$ The elements of $W$ are obtained by applying (3.11), being careful to convert from plane-wave to $S$-wave external states. Equations (3.22), (3.24) and (3.26) give the absorptive part of the non-relativistic amplitudes, which should be set equal to the corresponding relativistic amplitudes using the appropriate combinations of (3.12) and (3.14). Neglecting power corrections,

$$
\begin{aligned}
w_{ \pm} & =-\frac{\pi \alpha^{2}}{M^{2}}\left\{1+\frac{\alpha s_{W}^{-2}}{4 \pi}\left[-16 \log ^{2} \frac{m_{W}}{2 M}-8 \log \frac{m_{W}}{2 M}+\frac{3 \pi^{2}}{2}-18\right]\right\}+\mathcal{O}\left(\alpha^{4}, m_{W} / M\right) \\
w_{ \pm ; 00} & =-\frac{\pi \alpha^{2}}{M^{2}} \frac{\alpha s_{W}^{-2}}{4 \pi}\left[(8-8 i \pi) \log \frac{m_{W}}{2 M}-2-\frac{\pi^{2}}{2}\right]+\mathcal{O}\left(\alpha^{4}, m_{W} / M\right) \\
w_{00} & =-\frac{\pi \alpha^{2}}{M^{2}}\left(\frac{\alpha s_{W}^{-2}}{4 \pi}\right)^{2}\left[\left(8 \log \frac{m_{W}}{2 M}-2-\frac{\pi^{2}}{2}\right)^{2}+64 \pi^{2} \log ^{2} \frac{m_{W}}{2 M}\right]+\mathcal{O}\left(\alpha^{5}, m_{W} / M\right) .
\end{aligned}
$$

Note the presence of the $\log ^{2}\left(m_{W} / 2 M\right)$ factor (and its large coefficient) in the one-loop correction to $w_{ \pm}$. This large perturbative correction results in a numerically large suppression of WIMP cross sections compared to tree-level predictions, and signals a breakdown of perturbation theory for TeV scale WIMP masses.

Power corrections in $m_{W} / M$ to the matching coefficients $w_{i j}$ may be obtained by expanding the amplitudes (3.12), (3.14). In the $M \gtrsim \mathrm{TeV}$ mass regime, these corrections are numerically subleading compared to logarithmically enhanced perturbative corrections at leading power [71].

\subsection{Fixed order results}

Armed with the Sommerfeld matrix $s_{i j}$, and the elements of the $W$ matrix given in (3.27), we are in a position to compute the dark matter annihilation cross section to photons at both tree level (by simply truncating the $\alpha$ expansion in (3.27)) and one loop. The results of these two calculations are shown in figure 4 , where we have taken $\delta=0.17 \mathrm{GeV}$ and the relative velocity $v=10^{-3}$ in the numerical evaluation of the Sommerfeld enhancement. Clearly the one-loop result is suppressed with respect to the tree-level result. Specifically, we find that at $M=3 \mathrm{TeV}$ (a mass of interest for the thermal wino), the ratio $\sigma_{\text {tree }} / \sigma_{1 \text {-loop }} \sim 5$. However the perturbative expansion is not under control, as seen from the fact that the fixed order $\alpha^{3}$ cross section becomes negative for $M \gtrsim 6 \mathrm{TeV}$ due to the large Sudakov logarithm. The orange dot-dashed line gives the naive cross section computed from $w_{00}$ neglecting the Sommerfeld enhancement.

These considerations demonstrate the need for an EFT description that separates the scales $m_{W}$ and $M$, and resums large logarithms. The first step will be to derive an appropriate EFT description that captures all of the relevant momentum regions of the full theory. This is the topic of the next section.

\footnotetext{
${ }^{7}$ The source of the minus sign is simply that in the Lagrangian, $L=-V$, while the scattering matrix is conventionally defined as $T=+V+\ldots$.
} 


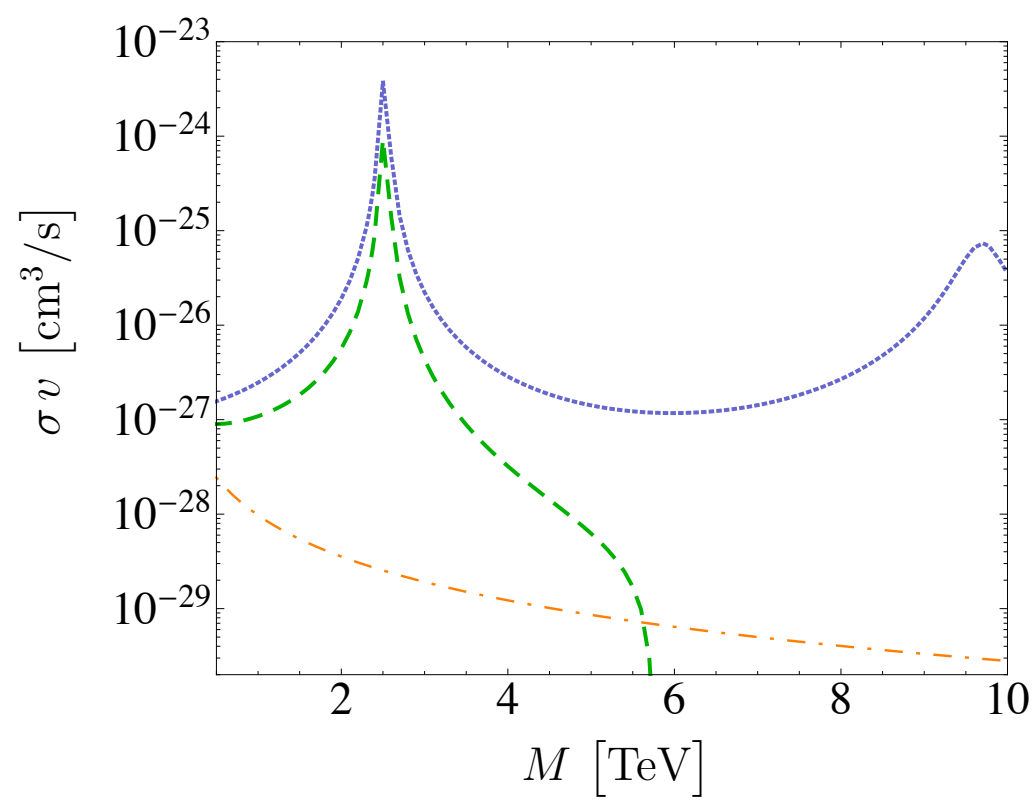

Figure 4. Sommerfeld enhanced annihilation cross sections for two fixed order approximations. The blue dotted curve truncates the $w$ factors at $O\left(\alpha^{2}\right)$, while the green dashed line is the one-loop result including $O\left(\alpha^{3}\right)$ contributions in $w_{ \pm}$and $w_{ \pm ; 00}$ and the first non-vanishing $O\left(\alpha^{4}\right)$ contribution in $w_{00}$. Note that for $M \gtrsim 6 \mathrm{TeV}$, the one-loop cross section becomes negative due to the presence of a large Sudakov logarithm with a negative coefficient. For illustration we include the orange dot-dashed line which gives the naive cross section computed from $w_{00}$ neglecting wavefunction enhancements. In this plot $v=10^{-3}$ and $\delta=0.17 \mathrm{GeV}$.

\section{Deriving the effective theory}

In the interesting regime of large WIMP mass, the cross section becomes uncertain due to large Sudakov logarithms, $\sim \alpha \log ^{2}\left(m_{W} / 2 M\right)$. We wish to develop an EFT framework that will isolate these enhanced contributions and systematically reorganize the perturbative expansion to resum them. The framework will also reveal certain universal features, including properties that are independent of the WIMP's spin or electroweak gauge representation, and simplify matching calculations at the hard scale $\mu \sim 2 M$ and weak scale $\mu \sim m_{W}$; e.g., the hard matching can be performed using electroweak symmetric Feynman rules.

This problem shares some features with processes involving electroweak vector boson production at colliders. However, one important difference is the presence of a heavy gauge-charged initial state in addition to jets of collinear charged final states, in contrast to the simpler Sudakov problem involving gauge-singlet heavy particle production [78-81]. The problem also shares some features with heavy particle pair production such as $t \bar{t}$ at colliders, but with different gauge group $-\mathrm{SU}(2)_{W} \times \mathrm{U}(1)_{Y}$ in place of $\mathrm{SU}(3)_{c}$ - and additional considerations of electroweak symmetry breaking.

\subsection{Regions analysis}

Different fields in the (soft collinear) effective theory will correspond to different momentum modes for the various particles in the original theory. To derive the fields required 


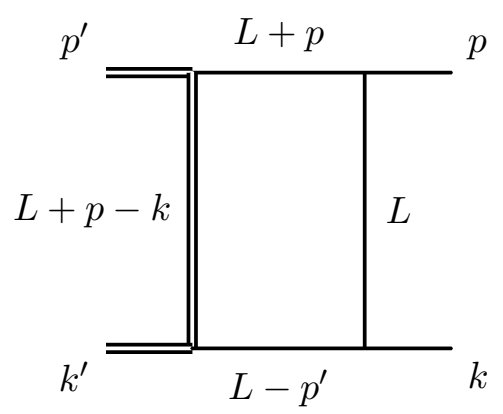

Figure 5. Diagrammatic representation of the integral (4.1). The double lines are propagators for a state with mass $M$, while the single lines are appropriate for massless particles.

to reproduce the IR structure of the full theory, we analyze the singularity structure of diagrams that contribute to heavy WIMP annihilation. This systematic decomposition of loop integrals is known as a regions analysis (for a monograph on this subject, see [82]). It simultaneously allows for the perturbative solution of the integrals when a separation of scales is present, while providing insight as to what modes are required to construct an EFT that can be matched to the full theory order-by-order in the gauge coupling and power counting parameter $\lambda=m_{W} / M$.

For concreteness, let us consider, e.g., the integral

$$
I=\int(d L) \frac{1}{L^{2}} \frac{1}{(L+p)^{2}} \frac{1}{\left(L-p^{\prime}\right)^{2}} \frac{1}{(L+p-k)^{2}-M^{2}} .
$$

Apart from numerator structure (inessential for the regions analysis), this integral corresponds to the diagram in figure 5 . We use the shorthand notation,

$$
(d L)=\frac{d^{d} L}{(2 \pi)^{d}}
$$

and employ dimensional regularization with $d=4-2 \epsilon$ dimensions.

The physical process of interest involves initial state heavy particles at rest annihilating to massless energetic particles. It is therefore useful to introduce the timelike unit vector $v^{\mu}$ with $v^{2}=1$, and lightcone vectors $n^{\mu}$ and $\bar{n}^{\mu}$ satisfying $n^{2}=\bar{n}^{2}=0$ and $n \cdot \bar{n}=2$. For momenta in the $\pm \hat{z}$ direction, a convenient choice is $n^{\mu}=(1,0,0,1), \bar{n}^{\mu}=(1,0,0,-1)$. While allowing a more general relation is convenient for some purposes (such as analyzing Lorentz invariance constraints of subleading corrections as done in [83, 84]), for simplicity we take $2 v=n+\bar{n}$. We take the heavy WIMPs to have momentum

$$
k=k^{\prime}=M v .
$$

For the massless final state particles, it is convenient to expand their momenta in lightcone components,

$$
p^{\mu} \longleftrightarrow\left(n \cdot p, \bar{n} \cdot p, p_{\perp}\right)
$$

where $p_{\perp}^{\mu}=p^{\mu}-(n \cdot p) \bar{n}^{\mu} / 2-(\bar{n} \cdot p) n^{\mu} / 2$. Let us consider the integral representing an amplitude with (offshell) final state momenta $p^{2} \sim p^{\prime 2} \sim M^{2} \lambda$, where $\lambda$ is the dimensionless 
power counting expansion parameter of SCET. For example, writing

$$
p=M n+\delta p, \quad p^{\prime}=M \bar{n}-\delta p
$$

such that $p+p^{\prime}=2 M v$, we may take $\delta p^{\mu}=\delta p_{\perp}^{\mu}$ so that $p^{2}=p^{\prime 2}=(\delta p)^{2} \sim M^{2} \lambda$. Evaluating the integral (4.1) in the limit $p^{2} / M^{2} \sim \lambda \ll 1$, we have

$$
I=\frac{\left[c_{\epsilon}\right]}{M^{4}}\left[-\frac{1}{4} \log ^{2}\left(\frac{-p^{2}+i 0}{4 M^{2}}\right)-\frac{\pi^{2}}{48}-\frac{i \pi}{4} \log \left(\frac{-p^{2}+i 0}{4 M^{2}}\right)+\mathcal{O}\left(p^{2} / M^{2}\right)\right]
$$

Consider the following momentum regions (decomposed along the light cone):

$$
\begin{array}{rll}
\text { (hard) } & \mathrm{h}: & L^{\mu} \sim M(1,1,1), \\
\text { (soft) } & s: & L^{\mu} \sim M(\lambda, \lambda, \lambda), \\
\text { (hardcollinear) } & \mathrm{hc}: & L^{\mu} \sim M\left(\lambda, 1, \lambda^{\frac{1}{2}}\right), \\
\text { (anti-hardcollinear) } & \overline{\mathrm{hc}}: & L^{\mu} \sim M\left(1, \lambda, \lambda^{\frac{1}{2}}\right) .
\end{array}
$$

Now we will show that these regions are sufficient to reproduce the full theory result, to leading order in $\lambda$. Taylor expanding the four-momentum $L$ in each denominator of (4.1) following the scalings in (4.7) gives the integrals

$$
=\int(d L) \frac{1}{L^{2}} \frac{1}{L^{2}+2 M n \cdot L} \frac{1}{L^{2}-2 M \bar{n} \cdot L} \frac{1}{L^{2}+M(n \cdot L-\bar{n} \cdot L)-2 M^{2}},
$$

There are also contributions from the momentum routing where the lines with $L+p$ and $L-p^{\prime}$ in figure 5 become soft. With momentum $L$ for the soft line,

$$
\begin{aligned}
& I_{s, \mathrm{hc}}=\int(d L) \frac{1}{p^{2}-2 M n \cdot L} \frac{1}{L^{2}} \frac{1}{4 M^{2}} \frac{1}{-M(n \cdot L+\bar{n} \cdot L)}, \\
& I_{s, \overline{\mathrm{hc}}}=\int(d L) \frac{1}{p^{2}+2 M \bar{n} \cdot L} \frac{1}{4 M^{2}} \frac{1}{L^{2}} \frac{1}{M(n \cdot L+\bar{n} \cdot L)} .
\end{aligned}
$$


An explicit evaluation of these integrals yields

$$
\begin{aligned}
I_{\mathrm{h}} & =M^{-2 \epsilon}\left[-\frac{1}{4 \epsilon^{2}}+\frac{1}{\epsilon}\left(\frac{1}{2} \log 2-\frac{i \pi}{8}\right)+\frac{5 \pi^{2}}{48}-\frac{1}{2} \log ^{2} 2+\frac{i \pi}{4} \log 2\right], \\
I_{s} & =\left(-\frac{p^{2} p^{2}}{4 M^{2}}+i 0\right)^{-\epsilon}\left(-\frac{1}{8 \epsilon^{2}}-\frac{\pi^{2}}{48}\right), \\
I_{\mathrm{hc}} & =\left(-p^{2}-i 0\right)^{-\epsilon}\left(\frac{1}{4 \epsilon^{2}}-\frac{\pi^{2}}{24}\right), \\
I_{\overline{\mathrm{hc}}} & =\left(-p^{2}-i 0\right)^{-\epsilon}\left(\frac{1}{4 \epsilon^{2}}-\frac{\pi^{2}}{24}\right) \\
I_{s, \mathrm{hc}} & =\left(-\frac{p^{2}}{2 M}-i 0\right)^{-2 \epsilon}\left(-\frac{1}{16 \epsilon^{2}}-\frac{\pi^{2}}{24}\right), \\
I_{s, \overline{\mathrm{hc}}} & =\left(-\frac{p^{2}}{2 M}-i 0\right)^{-2 \epsilon}\left(-\frac{1}{16 \epsilon^{2}}-\frac{\pi^{2}}{24}\right),
\end{aligned}
$$

where overall factors of $M^{-4}$ and $\left[c_{\epsilon}\right]=i(4 \pi)^{-2+\epsilon} \Gamma(1+\epsilon)$ have been dropped for simplicity. One can verify that the integrals in (4.10) sum to the expression (4.6) for the original integral (4.1).

This demonstrates the field content required for a complete EFT description of the diagram in figure 5 . The hard region $L^{\mu} \sim M(1,1,1)$ will be captured entirely in the Wilson coefficient of the annihilation operator in the EFT through matching at the scale $\mu \sim 2 M$. Then the contributions from IR modes (at leading power $\lambda^{0}$ ) are reproduced by momentum scalings in (4.7) that we identify with "hardcollinear" and "soft" regions. A Lagrangian field theory with fields corresponding to these modes can be constructed. The Feynman rules of this EFT will correspond to the explicit classes of diagrams of (4.8) and (4.9), thereby encoding the entire IR structure of the full theory as a controlled expansion in an explicit small parameter.

A similar analysis can be used to demonstrate that other diagrams are reproduced in the same manner by the sum over momentum regions of dimensionally regulated integrals. For the matching at the weak scale $\mu \sim m_{W}$, we may perform a similar analysis to isolate contributions from the potential region contained in the soft region of the diagrams. Potential regions have changed momenta scaling as $v \cdot L \sim M \lambda^{2}$ and $\left(L^{\mu}-v^{\mu} v \cdot L\right) \sim M \lambda$, and are resummed as the Sommerfeld enhancement by solving the quantum mechanical Hamiltonian.

This procedure allows for the systematic factorization of momentum regions: the scale $M$ will only appear in the hard matching coefficient (up to the collinear anomaly discussed in section 7.3 below), and the EFT will only depend on the IR scale $m_{W}$. By evolving the Wilson coefficients from $\mu \sim 2 M$ to $m_{W}$ using the RGEs of the EFT, the large logarithms discussed above are resummed, thereby systematically improving perturbation theory. The rest of this section provides the explicit construction of this EFT for application to heavy WIMP annihilation.

\subsection{Heavy particle and soft collinear effective theory for WIMP annihilation}

Having motivated the introduction of soft, hardcollinear and anti-hardcollinear modes, we now proceed to construct an effective theory describing interactions at scales $m_{W} \ll \mu \ll$ 
$M$. We perform this analysis in the electroweak symmetric vacuum; accounting for the effects of electroweak symmetry breaking will be discussed in section 7 below.

We focus for simplicity on a self-conjugate scalar WIMP, necessarily a U(1) $Y$ hypercharge singlet that transforms under a general integer isospin representation of $\mathrm{SU}(2)_{W}$, with generators $t^{a}$. We ignore Standard Model field content beyond the $\mathrm{SU}(2)_{W}$ gauge fields; modifications to this case are straightforward. In the absence of collinear degrees of freedom, the heavy WIMP is described as a heavy particle field, with Lagrangian [31]

$$
\mathcal{L}_{\phi_{v}}=\phi_{v}^{*}(i v \cdot D+\ldots) \phi_{v},
$$

where $\phi_{v}$ denotes the scalar heavy particle field, $v^{\mu}$ is the heavy particle velocity introduced above, and $D_{\mu}$ is the $\mathrm{SU}(2)_{W}$ covariant derivative (2.2).

The soft, hardcollinear and anti-hardcollinear gauge fields are denoted by $A_{s}^{\mu}, A_{\mathrm{hc}}^{\mu}$ and $A_{\mathrm{hc}}^{\mu}$, and are described by respective Lagrangians that are formally identical to those for the full $\mathrm{SU}(2)_{W}$ gauge theory, with the understanding that each field is restricted to the appropriate momentum mode. We suppress the matrix structure, $A_{\mu} \equiv A_{\mu}^{a} t^{a}$, and, to avoid conflicting notation with Wilson lines below, denote the $\mathrm{SU}(2)_{W}$ gauge field by $A_{\mu}$ (instead of $W_{\mu}$ ). Corresponding to the scalings in (4.7), a power counting in which the gauge field components scale in the same way as their momentum is assigned:

$$
A_{s}^{\mu} \sim(\lambda, \lambda, \lambda), \quad A_{\mathrm{hc}}^{\mu} \sim\left(\lambda, 1, \lambda^{\frac{1}{2}}\right), \quad \text { and } \quad A_{\mathrm{hc}}^{\mu} \sim\left(1, \lambda, \lambda^{\frac{1}{2}}\right) .
$$

In this way, Lagrangian interactions may be expanded as a series in $\lambda$. Amputated Feynman diagrams and corresponding $S$ matrix elements will obey a simple power counting based on the appearance of the associated vertices [52, 53, 57-59]. Gauge fixing and ghosts can be treated in the standard way.

This power counting implies that leading order interactions may occur between soft and hardcollinear fields (or between soft and anti-hardcollinear fields), since, e.g. $n \cdot A_{s} \sim$ $n \cdot A_{\mathrm{hc}} \sim \lambda$. At leading order, the interactions of the soft field with the hardcollinear sector are given by the replacement in the hardcollinear Lagrangian,

$$
A_{\mathrm{hc}}^{\mu}(x) \rightarrow A_{\mathrm{hc}}^{\mu}(x)+n \cdot A_{s}\left(x_{-}\right) \frac{\bar{n}^{\mu}}{2},
$$

where $x_{-}^{\mu} \equiv(\bar{n} \cdot x) n^{\mu} / 2$ and $x_{+}^{\mu} \equiv(n \cdot x) \bar{n}^{\mu} / 2$ are arbitrary four-vectors expanded along the light cone. The "multipole" expansion of $A_{s}(x)=A_{s}\left(x_{-}\right)+\mathcal{O}\left(\lambda^{2}\right)$ ensures that only the $n \cdot p_{s}$ components of soft momenta are added to hardcollinear momenta. Similar considerations, with $n \leftrightarrow \bar{n}$, apply to the interactions between soft and anti-hardcollinear fields.

The local gauge invariance of the full theory is mapped to separate soft, hardcollinear and anti-hardcollinear gauge transformations in the effective theory,

$$
\begin{aligned}
& s: \quad A_{\mathrm{hc}}^{\mu} \rightarrow V_{s}\left(x_{-}\right) A_{\mathrm{hc}}^{\mu} V_{s}^{\dagger}\left(x_{-}\right), \quad A_{\mathrm{hc}}^{\mu} \rightarrow V_{s}\left(x_{+}\right) A_{\mathrm{hc}}^{\mu} V_{s}^{\dagger}\left(x_{+}\right), \\
& A_{s}^{\mu} \rightarrow V_{s} A_{s}^{\mu} V_{s}^{\dagger}+\frac{i}{g} V_{s}\left[\partial^{\mu}, V_{s}^{\dagger}\right] \\
& \text { hc }: \quad A_{\mathrm{hc}}^{\mu} \rightarrow V_{\mathrm{hc}} A_{\mathrm{hc}}^{\mu} V_{\mathrm{hc}}^{\dagger}+\frac{i}{g} V_{\mathrm{hc}}\left[\partial^{\mu}-i g A_{s,+}^{\mu}\left(x_{-}\right), V_{\mathrm{hc}}^{\dagger}\right], \quad A_{\mathrm{hc}}^{\mu} \rightarrow A_{\mathrm{hc}}^{\mu}, \quad A_{s}^{\mu} \rightarrow A_{s}^{\mu} \text {, } \\
& \overline{\mathrm{hc}}: \quad A_{\mathrm{hc}}^{\mu} \rightarrow A_{\mathrm{hc}}^{\mu}, \quad A_{\overline{\mathrm{hc}}}^{\mu} \rightarrow V_{\overline{\mathrm{hc}}} A_{\overline{\mathrm{hc}}}^{\mu} V_{\overline{\mathrm{hc}}}^{\dagger}+\frac{i}{g} V_{\overline{\mathrm{hc}}}\left[\partial^{\mu}-i g A_{s,-}^{\mu}\left(x_{+}\right), V_{\overline{\mathrm{hc}}}^{\dagger}\right], \quad A_{s}^{\mu} \rightarrow A_{s}^{\mu} .
\end{aligned}
$$


With these preliminaries, we can determine the leading order basis of operators representing heavy WIMP annihilation to di-boson final states. Since components of the derivatives and gauge fields count as $\mathcal{O}(1)$ in the power counting, e.g., $\bar{n} \cdot A_{\mathrm{hc}} \sim 1$, operators are built from field combinations that implement lightcone gauges $\bar{n} \cdot \mathcal{A}_{\mathrm{hc}}=n \cdot \mathcal{A}_{\overline{\mathrm{hc}}}=0$. Expressed in an arbitrary gauge, these fields read,

$$
g \mathcal{A}_{\mathrm{hc}}^{\mu}=W^{\dagger} i D_{\mathrm{hc}}^{\mu} W, \quad g \mathcal{A}_{\mathrm{hc}}^{\mu}=\bar{W}^{\dagger} i D_{\overline{\mathrm{hc}}}^{\mu} \bar{W},
$$

where $i D_{\mathrm{hc}(\overline{\mathrm{hc}})}^{\mu}=i \partial^{\mu}+g\left(A_{\mathrm{hc}(\overline{\mathrm{hc}})}^{\mu}+A_{s \pm}\left(x_{\mp}\right)^{\mu}\right)$, and $W(\bar{W})$ is a Wilson line of hardcollinear (anti-hardcollinear) fields in the $\bar{n}(n)$ direction,

$$
W(x)=\mathrm{P} \exp \left[i g \int_{-\infty}^{0} d s \bar{n} \cdot A_{\mathrm{hc}}(x+s \bar{n})\right], \quad \bar{W}(x)=\mathrm{P} \exp \left[i g \int_{-\infty}^{0} d s n \cdot A_{\overline{\mathrm{hc}}}(x+s n)\right] .
$$

Noting the scaling relations,

$$
\mathcal{A}_{\mathrm{hc}}^{\mu} \sim\left(\lambda, 0, \lambda^{\frac{1}{2}}\right), \quad \mathcal{A}_{\mathrm{hc}}^{\mu} \sim\left(0, \lambda, \lambda^{\frac{1}{2}}\right)
$$

we see that operators mediating leading-order processes with two initial state heavy WIMPs, one final state hardcollinear field and one final state anti-hardcollinear field are of the form $\phi_{v}^{a} \phi_{v}^{b} \mathcal{A}_{\mathrm{hc} \perp}^{c \mu} \mathcal{A}_{\mathrm{hc} \perp}^{d \nu}$, with gauge indices $a, b, c, d$ contracted to form invariant combinations. It is straightforward to see that, for an arbitrary $\mathrm{SU}(2)_{W}$ representation, there are two such operators,

$$
\mathcal{L}_{\phi_{v} \phi_{v}}=\frac{1}{M} \sum_{i=1}^{2} c_{i} O_{i}+\text { h.c. }+\mathcal{O}\left(1 / M^{2}\right)
$$

where the explicit form of these dimension 5 operators is given by

$$
\begin{aligned}
& O_{1}=g^{2} \phi_{v}^{T} \phi_{v} \mathcal{A}_{\mathrm{hc} \perp \mu}^{a} \mathcal{A}_{\overline{\mathrm{hc}} \perp}^{a}{ }^{\mu}=g^{2} \phi_{v}^{i} \phi_{v}^{j} \mathcal{A}_{\mathrm{hc} \mu}^{a} \mathcal{A}_{\overline{\mathrm{hc}} \nu}^{b} \delta^{i j} \delta^{a b} g_{\perp}^{\mu \nu} \\
& O_{2}=g^{2} \phi_{v}^{T} \mathcal{A}_{\mathrm{hc} \perp \mu} \mathcal{A}_{\overline{\mathrm{hc}} \perp}{ }^{\mu} \phi_{v}=g^{2} \phi_{v}^{i} \phi_{v}^{j} \mathcal{A}_{\mathrm{hc} \mu}^{a} \mathcal{A}_{\overline{\mathrm{hc}} \nu}^{b}\left(t^{a} t^{b}\right)^{i j} g_{\perp}^{\mu \nu} .
\end{aligned}
$$

Here $g_{\perp}^{\mu \nu}=g^{\mu \nu}-\left(n^{\mu} \bar{n}^{\nu}+\bar{n}^{\mu} n^{\nu}\right) / 4$ projects onto transverse components. Note that the coupling factor $g^{2}$ is included in the operator definition (as opposed to being absorbed into $c_{i}$ ) for convenience in the renormalization analysis.

In the following, we consider the matching of full theory amplitudes at the hard scale $\mu \sim 2 M$, and evolve the resulting matching coefficients to the scale $\mu \sim m_{W}$ by computing the anomalous dimensions and solving the evolution equation.

\subsection{Electroweak symmetric SCET Feynman rules}

In this section, we give the Feynman rules for the effective theory describing interactions at scales $m_{W} \ll \mu \ll M$. Note that the presence of the hardcollinear gauge boson operators $\mathcal{A}$ in the definition of the $O_{i}$ implies that there can be an arbitrary number of gauge 
boson emissions from the operator vertex insertion. We use 't Hooft-Feynman gauge in the following. The Feynman rules for operator insertions of $O_{1,2}$ are:

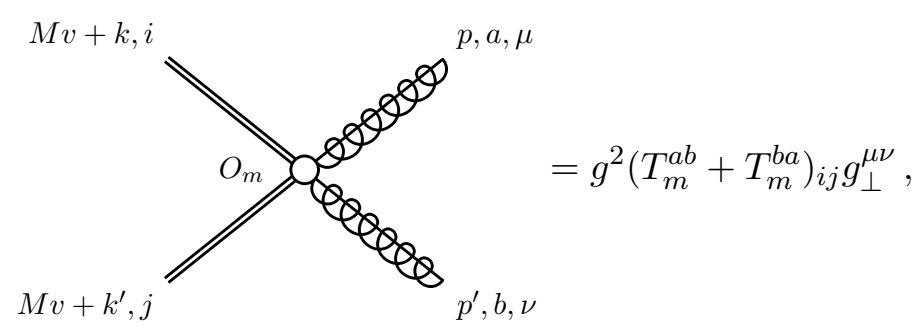

where the color structures, defined as

$$
\left(T_{1}^{a b}\right)_{i j}=\delta^{a b} \delta_{i j}, \quad\left(T_{2}^{a b}\right)_{i j}=\left(t^{a} t^{b}\right)_{i j}
$$

are taken from (4.19). Note that this involves one hardcollinear (top of diagram) and one anti-hardcollinear (bottom of diagram) particle. We need also the Feynman rule with an additional hardcollinear, or anti-hardcollinear gauge boson from the operator vertex. The Feynman rule for two hardcollinear and one anti-hardcollinear emissions is

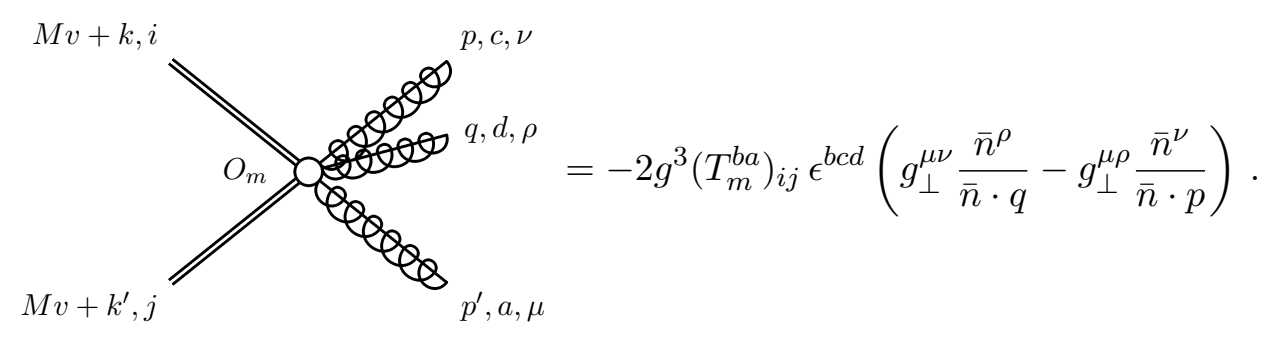

Similar expressions, with $n \leftrightarrow \bar{n}$, hold for one hardcollinear and two anti-hardcollinear emissions. The three- and four-point vertices involving all hardcollinear or all anti-hardcollinear gauge bosons are identical to the usual QCD results. As in (4.13), the leading order interaction of soft gauge bosons with hardcollinear gauge bosons is given by the multipole expansion in powers of $\lambda$ of

$$
\mathcal{L}_{\mathrm{hc}, s}=\frac{g}{2} \epsilon^{a b c} n \cdot A_{s}^{c} A_{\mathrm{hc} \mu}^{a}\left(2 \partial^{\mu} \bar{n} \cdot A_{\mathrm{hc}}^{b}-\bar{n} \cdot \partial A_{\mathrm{hc}}^{b \mu}\right)+\ldots,
$$

yielding the Feynman rule (here all momenta are ingoing)

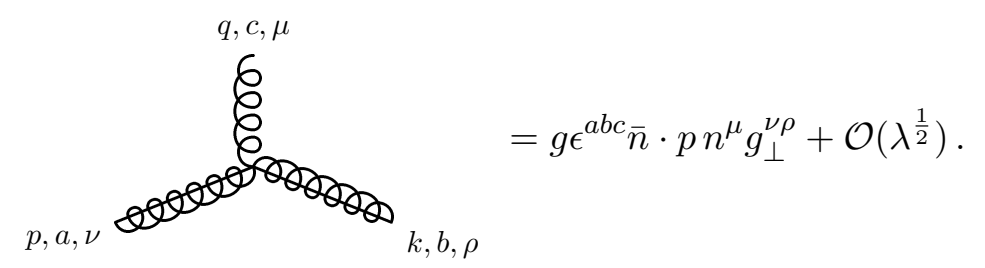

The interaction of soft gauge bosons with heavy scalars is given by the usual result,

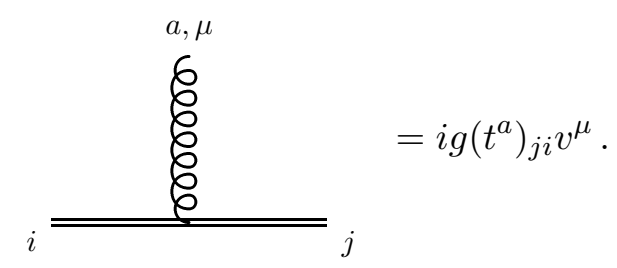


Armed with these Feynman rules, the renormalized Wilson coefficients $c_{i}(\mu)$ can be computed by matching the full theory onto the EFT at $\mu \sim 2 M$ (the subject of section 5 ). Furthermore, anomalous dimensions for the operators $O_{i}$ can be computed, which determine the RGEs that allow us to compute $c_{i}\left(m_{W}\right)$ using $c_{i}(2 M)$ as input (the subject of section 6).

\section{$5 \quad$ High scale matching}

This section provides the matching calculation between the electroweak symmetric full and effective theories at renormalization scale $\mu \sim 2 M \gg m_{W}$. Consider the process $\phi_{i}(k)+\phi_{j}\left(k^{\prime}\right) \rightarrow A^{a}(p)+A^{b}\left(p^{\prime}\right)$. Given two initial state WIMPs at zero velocity $k=k^{\prime}=$ $M v$, conservation of momentum implies that the massless final state gauge bosons have $p=M n$ and $p^{\prime}=M \bar{n}$. Therefore, all factors of $\bar{n} \cdot p$ and $n \cdot p^{\prime}$ will be replaced with $2 M$ in what follows.

\subsection{Matching conditions}

The matching condition can be stated as

$$
\frac{1}{2 M}\left(Z_{\phi, \text { full }}^{\frac{1}{2}}\right)^{2}\left(Z_{A, \text { full }}^{\frac{1}{2}}\right)^{2} \sum_{i} \mathcal{M}_{i, \text { full }} \frac{\left\langle O_{i}\right\rangle^{\text {tree }}}{g^{2}(\mu)}=\left(Z_{\phi_{v}}^{\frac{1}{2}}\right)^{2}\left(Z_{A, \text { eff }}^{\frac{1}{2}}\right)^{2} \sum_{i} c_{i}^{\text {bare }}\left\langle O_{i}^{\text {bare }}\right\rangle
$$

where the onshell wavefunction renormalization factors for the external particles ensure that we are comparing two physical amplitudes. The factor of $\left\langle O_{i}\right\rangle^{\text {tree }}$ on the left hand side accounts for color and polarization structures (see (4.20) for the explicit expression). We have here defined the tree-level matrix element without gauge coupling as

$$
\left\langle O_{i}\right\rangle^{\text {tree }} \equiv \frac{g^{2}(\mu)}{g_{\text {bare }}^{2}}\left\langle O_{i}^{\text {bare }}\right\rangle^{\text {tree }}
$$

We will solve (5.1) for the bare Wilson coefficients $c_{i}^{\text {bare }}$.

Since we are working with electroweak symmetric SCET, there are no dimensionful parameters in the theory. Noting that scaleless integrals are zero in dimensional regularization, the effective theory loop integrals and renormalization factors vanish. Hence,

$$
\left(Z_{\phi_{v}}^{\frac{1}{2}}\right)^{2}\left(Z_{A, \text { eff }}^{\frac{1}{2}}\right)^{2} \sum_{i} c_{i}^{\text {bare }}\left\langle O_{i}^{\text {bare }}\right\rangle=Z_{g}^{2} \mu^{2 \epsilon} \sum_{i} c_{i}^{\text {bare }}\left\langle O_{i}\right\rangle^{\text {tree }} .
$$

It is straightforward to identify the bare matching coefficients with the corresponding full theory diagrams using (5.1).

The amputated full theory diagrams are depicted in figure 6. Note that real emissions from the initial state heavy WIMPs, and the associated vertex corrections, are power suppressed. Real emissions from the final state bosons are relevant for the $W^{+} W^{-}$annihilation channel, and is left to future work [71]. In terms of the bare coupling constant $\tilde{g}_{\text {bare }}$ 


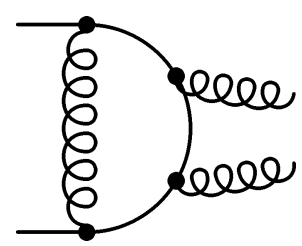

(a)

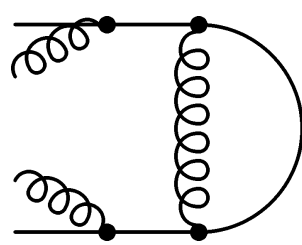

(e)

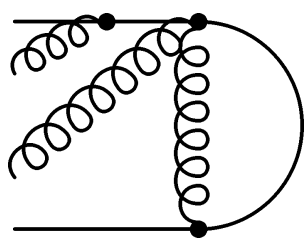

(i)

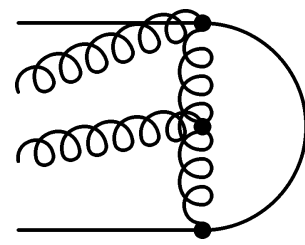

$(\mathrm{m})$

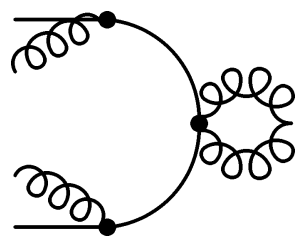

(q)

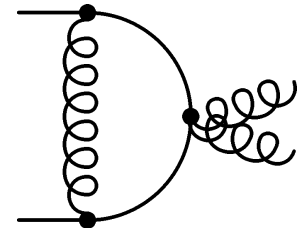

(b)

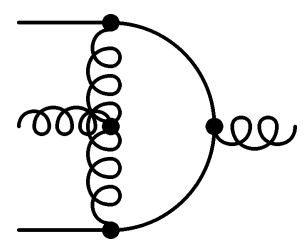

(f)

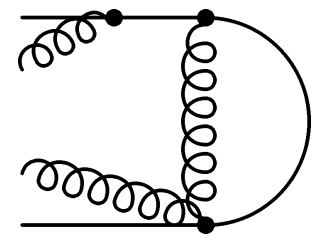

(j)

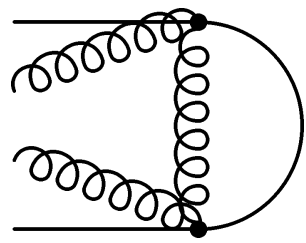

(n)

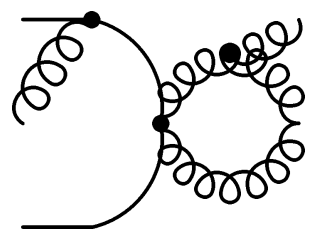

(r)

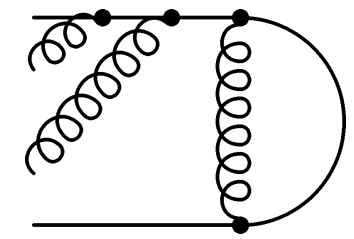

(c)

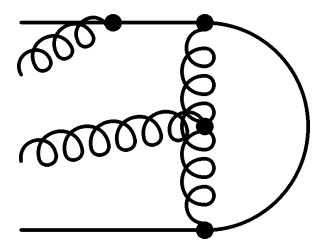

(g)

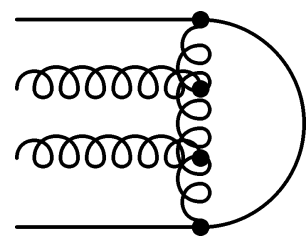

(k)

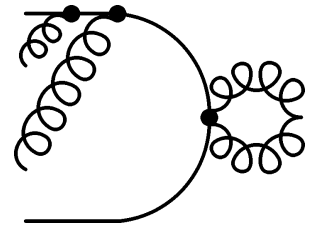

(o)

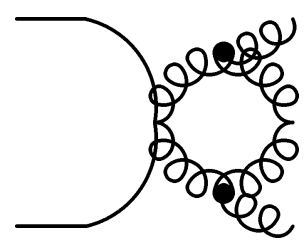

(s)

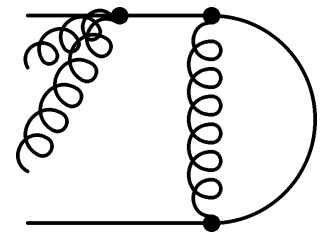

(d)

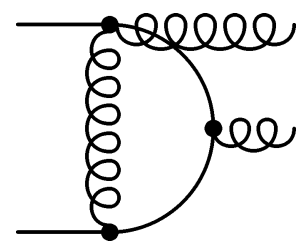

(h)

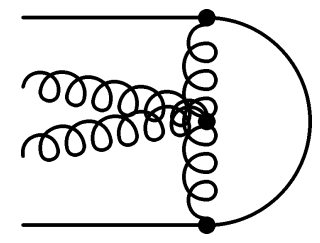

(l)

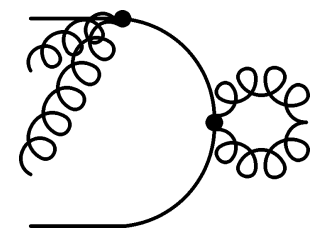

(p)

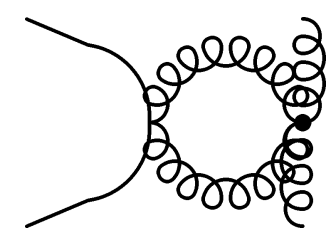

(t)

Figure 6. Diagrams contributing to hard scale matching.

of the full theory, the resulting amplitudes read,

$$
\begin{aligned}
\mathcal{M}_{1, \text { full }}= & -i\left[c_{\epsilon}\right] \tilde{g}_{\text {bare }}^{4}(2 M)^{-2 \epsilon}\left\{\frac{1}{\epsilon}(2-2 i \pi) C_{2}(j)+C_{2}(j)\left(-1-\frac{\pi^{2}}{4}\right)\right\}, \\
\mathcal{M}_{2, \text { full }}= & \tilde{g}_{\text {bare }}^{2}-i\left[c_{\epsilon}\right] \tilde{g}_{\text {bare }}^{4}(2 M)^{-2 \epsilon} \\
& \times\left\{-\frac{4}{\epsilon^{2}}+\frac{1}{\epsilon}[-6+2 i \pi]+C_{2}(j)\left(-7-\frac{\pi^{2}}{4}\right)+7+\frac{29 \pi^{2}}{12}\right\},
\end{aligned}
$$

where $C_{2}(j)=j(j+1)$ is the quadratic Casimir invariant for the spin- $j$ representation of 
$\mathrm{SU}(2)_{W}$. Note that we distinguish $\tilde{g}$ and $\tilde{Z}_{g}$ in the full theory from $g$ and $Z_{g}$ in the effective theory, which differ because the heavy WIMP has been integrated out below the scale $M$ and as such no longer contributes to the running of the gauge coupling. Specifically, at one loop the relation between $g$ and $\tilde{g}$ is [85]

$$
\frac{\tilde{g}^{2}}{g^{2}}=1-\frac{C(j)}{3} \frac{g^{2}}{(4 \pi)^{2}} \log \frac{M}{\mu} .
$$

Working in 't Hooft-Feynman gauge, the onshell wavefunction renormalization factors for the full theory fields in the electroweak symmetric vacuum can be derived at one loop to be

$$
\begin{aligned}
& Z_{\phi, \text { full }}=1 \\
& Z_{A, \text { full }}=1+\frac{\tilde{g}^{2}}{(4 \pi)^{2}}\left(\frac{M}{\mu}\right)^{-2 \epsilon}\left[-\frac{1}{6 \epsilon} C(j)+\mathcal{O}(\epsilon)\right]
\end{aligned}
$$

where $\operatorname{tr}\left(t^{c} t^{d}\right) \equiv C(j) \delta^{c d}$, so that $C(j)=j(j+1)(2 j+1) / 3$. Note that only the heavy WIMP contributes to $Z_{A \text {,full }}$ since the Standard Model matter is massless and therefore the corresponding integrals are zero in dimensional regularization. In the final result for renormalized hard coefficients, the finite term in $Z_{A, \text { full }}$ cancels with the contribution from the decoupling relation in (5.5). To relate $\tilde{g}^{\text {bare }}$ in (5.4) to $\tilde{g}(\mu)$ and hence $g(\mu)$ in (5.5), we require

$$
\tilde{g}_{\mathrm{bare}}=\tilde{Z}_{g} \mu^{\epsilon} \tilde{g}(\mu), \quad \tilde{Z}_{g}=1+\frac{\tilde{g}^{2}}{(4 \pi)^{2}} \frac{1}{\epsilon}\left[\frac{1}{12} C(j)-\frac{43}{12}+\frac{2}{3} n_{G}\right]
$$

where we have included $n_{G}=3$ generations of Standard Model fermions, the Standard Model Higgs doublet, and the heavy scalar WIMP contributions. ${ }^{8}$

The bare coefficients $c_{i}^{\text {bare }}$ are obtained from (5.1), employing the results (5.4) and (5.6) for the full theory side of the matching condition. In the next section, we determine the counterterms in the EFT, such that the renormalized coefficients $c_{i}(\mu)$ of the effective Lagrangian (4.18) can be derived and used as input to the RGEs.

\subsection{Renormalized matching coefficients}

In this section, we renormalize $c_{i}^{\text {bare }}$. Given the Feynman rules for electroweak symmetric SCET provided in section 4.3 above, we can compute one-loop contributions to the matrix elements of $O_{i}$ between external states of two scalars and two hardcollinear gauge bosons. We regulate infrared divergences with offshell momenta $p$ and $p^{\prime}$ for the final state gauge bosons and $k$ and $k^{\prime}$ for the initial state heavy WIMPs. Note that this regulation introduces a scale, allowing us access to the UV divergent contributions to the one-loop diagrams, which in turn can be used to derive the counterterms needed to renormalize the theory.

\footnotetext{
${ }^{8}$ The one-loop correction from Standard Model fields is proportional to $-\frac{11}{3} C_{2}(j=1)+\frac{2}{3}\left[n_{G}\left(N_{c}+\right.\right.$ $1)] C(j=1 / 2)+\frac{1}{3} C(j=1 / 2)$, where the three terms correspond to $\mathrm{SU}(2)_{W}$ gauge bosons, Standard Model fermions with $n_{G}=3$ generations and $N_{c}=3$ colors, and a Higgs doublet, respectively. The $C(j) / 12$ term accounts for the scalar WIMP contribution. $\mathrm{R}$
} 


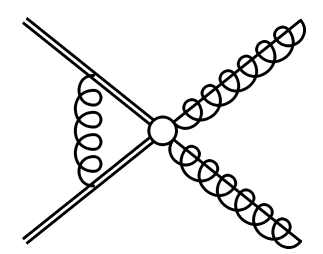

(a)

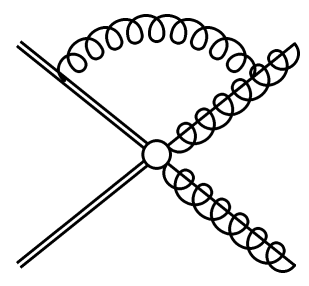

(e)

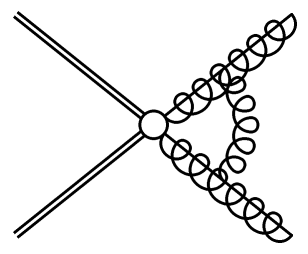

(b)

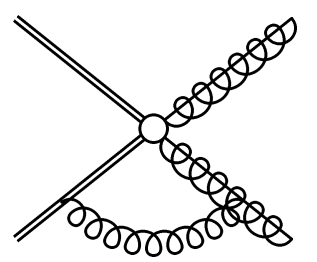

(f)

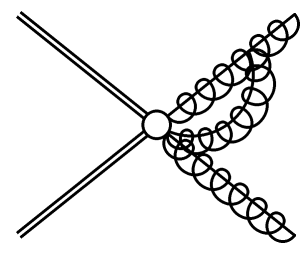

(c)

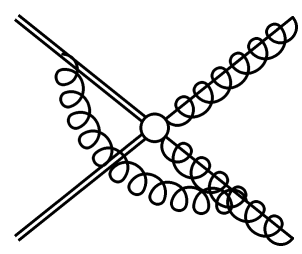

(g)

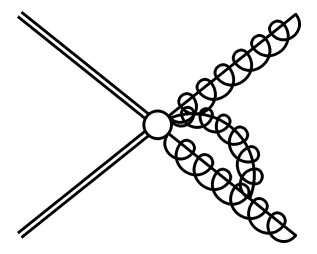

(d)

Figure 7. One-loop contributions to matrix elements of $O_{i}$.

We will need expressions for the renormalized gauge coupling, and wavefunction renormalization factors for the heavy scalar and gauge bosons in the EFT. Employing ' $t$ Hooft Feynman gauge, the one-loop expressions are

$$
g_{\text {bare }}=Z_{g} \mu^{\epsilon} g(\mu), \quad Z_{g}=1+\frac{g^{2}}{(4 \pi)^{2}} \frac{1}{\epsilon}\left[-\frac{43}{12}+\frac{2}{3} n_{G}\right],
$$

and

$$
Z_{\phi_{v}, \mathrm{EFT}}=1+\frac{g^{2}}{(4 \pi)^{2}} \frac{1}{\epsilon}\left[2 C_{2}(r)\right], \quad Z_{A, \mathrm{EFT}}=1+\frac{g^{2}}{(4 \pi)^{2}} \frac{1}{\epsilon}\left[\frac{19}{6}-\frac{4}{3} n_{G}\right],
$$

where in $Z_{A \text {,EFT }}$ we account for $n_{G}=3$ generations of Standard Model fermions, and the Standard Model Higgs doublet as in (5.7) above.

Evaluating the diagrams in figure 7 yields the UV divergences of the effective theory matrix elements. Multiplying by appropriate $Z$ factors to obtain physical $S$ matrix elements yields

$$
\begin{aligned}
& Z_{\phi_{v}, \mathrm{EFT}} Z_{A, \mathrm{EFT}}\left\langle O_{1}^{\text {bare }}\right\rangle \\
& =\left\langle O_{1}\right\rangle^{\text {tree }} Z_{g}^{2}\left\{1+\frac{g^{2}}{(4 \pi)^{2}}\left[\frac{4}{\epsilon^{2}}+\frac{1}{\epsilon}\left(\frac{43}{6}-\frac{4}{3} n_{G}-4 \log \frac{4 M^{2}}{\mu^{2}}+4 i \pi\right)\right]\right\} \\
& Z_{\phi_{v}, \mathrm{EFT}} Z_{A, \mathrm{EFT}}\left\langle O_{2}^{\text {bare }}\right\rangle \\
& =\left\langle O_{1}\right\rangle^{\text {tree }} Z_{g}^{2} \frac{g^{2}}{(4 \pi)^{2}} \frac{1}{\epsilon}\left[-2 C_{2}(r)+2 i \pi C_{2}(r)\right] \\
& \quad+\left\langle O_{2}\right\rangle^{\text {tree }} Z_{g}^{2}\left\{1+\frac{g^{2}}{(4 \pi)^{2}}\left[\frac{4}{\epsilon^{2}}+\frac{1}{\epsilon}\left(\frac{79}{6}-\frac{4}{3} n_{G}-4 \log \frac{4 M^{2}}{\mu^{2}}-2 i \pi\right)\right]\right\}
\end{aligned}
$$

from which we read off the operator renormalization matrix,

$$
\vec{O}^{\text {bare }}=\hat{Z} \vec{O}(\mu)
$$


where it is understood that $g^{\text {bare }}$ in $O_{i}^{\text {bare }}$ is expressed in terms of renormalized $g(\mu)$, and the factors $Z_{g}^{2} \mu^{2 \epsilon}$ are absorbed into $\hat{Z}$. The entries of the operator renormalization matrix are thus

$$
\begin{aligned}
& Z_{11}=1+\frac{g^{2}}{(4 \pi)^{2}}\left[\frac{4}{\epsilon^{2}}+\frac{1}{\epsilon}\left(-4 \log \frac{4 M^{2}}{\mu^{2}}+4 i \pi\right)\right], \\
& Z_{12}=0, \\
& Z_{21}=\frac{g^{2}}{(4 \pi)^{2}} \frac{1}{\epsilon}\left[-2 C_{2}(r)+2 i \pi C_{2}(r)\right], \\
& Z_{22}=1+\frac{g^{2}}{(4 \pi)^{2}}\left[\frac{4}{\epsilon^{2}}+\frac{1}{\epsilon}\left(6-4 \log \frac{4 M^{2}}{\mu^{2}}-2 i \pi\right)\right],
\end{aligned}
$$

where, as mentioned above, we have set $\bar{n} \cdot p=n \cdot p^{\prime}=2 M$ appropriate for the kinematics of interest.

Now we have all the ingredients necessary to derive the renormalized Wilson coefficients $c_{i}(\mu)$. Combining the expression for $c_{i}^{\text {bare }}$ derived from (5.1) with the result for operator renormalization (5.12), we obtain

$$
\vec{c}(\mu)=\hat{Z}^{T} \vec{c}^{\text {bare }} .
$$

Expressed in terms of the renormalized gauge coupling, the renormalized Wilson coefficients are given by

$$
\begin{gathered}
c_{1}(\mu)=\frac{1}{2} \frac{g^{2}}{(4 \pi)^{2}}\left[-(4-4 \pi i) C_{2}(r) \log \frac{2 M}{\mu}+C_{2}(r)\left(-1-\frac{\pi^{2}}{4}\right)\right]+\mathcal{O}\left(g^{4}\right), \\
c_{2}(\mu)=\frac{1}{2}\left\{1+\frac{g^{2}}{(4 \pi)^{2}}\left[-8 \log ^{2} \frac{2 M}{\mu}+(12-4 \pi i) \log \frac{2 M}{\mu}\right.\right. \\
\left.\left.+\left(-7-\frac{\pi^{2}}{4}\right) C_{2}(r)+7+\frac{29 \pi^{2}}{12}\right]\right\}+\mathcal{O}\left(g^{4}\right) .
\end{gathered}
$$

These are the hard scale matching coefficients. In the next section, the RGEs will be derived to evolve these coefficients down to the weak scale.

\section{Renormalization group evolution}

Robust predictions of the annihilation cross section for heavy WIMPs demand control over the Sudakov-type logarithms, e.g., appearing at $\mathcal{O}\left(e^{2} g_{2}^{2}\right)$ in the amplitude $\mathcal{M}^{+-\rightarrow \gamma \gamma}$ given in (3.14). In this section, we investigate the resummation of such large logarithms by solving the evolution of the coefficients $c_{i}(\mu)$ appearing in (4.18) from the hard annihilation scale $\mu_{H} \sim 2 M$ down to the electroweak scale $\mu_{L} \sim m_{W}$. The anomalous dimension for the basis of operators in (4.19) follows from renormalization properties of Wilson lines, and is given by an ansatz for the anomalous dimension of $n$-jet operators in SCET [44-47]. We illustrate the explicit connection between the universal cusp piece and the Sudakov double log, and present ingredients necessary for resummation through leading log (LL) and next-to-leading log (NLL) accuracy. 


\subsection{Anomalous dimensions}

The scale evolution of coefficients is governed by the RGE

$$
\frac{d}{d \log \mu} \vec{c}(\mu)=\hat{\Gamma}^{T} \vec{c}(\mu), \quad \hat{\Gamma}=\hat{Z}^{-1} \frac{d}{d \log \mu} \hat{Z}
$$

where $\hat{\Gamma}$ denotes the anomalous dimension matrix. With $\hat{Z}$ given in (5.12), we obtain

$$
\hat{\Gamma}=\frac{g^{2}}{(4 \pi)^{2}}\left(\begin{array}{cc}
8 \log \frac{4 M^{2}}{\mu^{2}}-8 i \pi & 0 \\
4 C_{2}(j)-4 i \pi C_{2}(j) & 8 \log \frac{4 M^{2}}{\mu^{2}}-12+4 i \pi
\end{array}\right)+\mathcal{O}\left(g^{4}\right)
$$

The logarithmic scaling of the diagonal elements is a universal feature related to the cusp anomalous dimension of Wilson loops, which can be identified as the origin of the large Sudakov logarithm in (3.14). The non-cusp part of the anomalous dimension depends on the gauge representations of the external states. It is convenient to rotate to a basis of operators with definite isospin $R=0$ and $R=2$, given respectively by $O_{1}^{\prime}=O_{1}$ and $O_{2}^{\prime}=2 O_{1} / 3-O_{2}$. In this basis the anomalous dimension is diagonal,

$$
\hat{\Gamma}^{\prime}=\frac{g^{2}}{(4 \pi)^{2}}\left(\begin{array}{cc}
8 \log \frac{4 M^{2}}{\mu^{2}}-8 i \pi & 0 \\
0 & 8 \log \frac{4 M^{2}}{\mu^{2}}-12+4 i \pi
\end{array}\right)+\mathcal{O}\left(g^{4}\right) .
$$

We may then identify $\hat{\Gamma}^{\prime}$ with an ansatz for the anomalous dimension of an operator describing a particle of mass $2 M$ in gauge representation $R$ decaying into two massless gauge bosons in gauge representations $r$ and $r^{\prime}$ [44-47],

$$
\begin{aligned}
\Gamma(R)= & \frac{1}{2} \gamma_{\text {cusp }}\left[\left(C_{2}(r)+C_{2}\left(r^{\prime}\right)\right)\left(\log \frac{4 M^{2}}{\mu^{2}}-i \pi\right)+i \pi C_{2}(R)\right] \\
& +\gamma^{r}+\gamma^{r^{\prime}}+\gamma^{R}-2 \frac{\beta(g)}{g} .
\end{aligned}
$$

This makes the connection with the cusp anomalous dimension $\gamma_{\text {cusp }}$ explicit. Note that the coefficient of $\log 4 M^{2} / \mu^{2}$ is independent of the WIMP's spin and quantum numbers, demonstrating the universality of the Sudakov suppression for heavy WIMP annihilation.

The term in (6.4) involving the beta function $\beta(g)=d g / d \log \mu$ appears due to the factor of $g^{2}$ in the operator definition. Employing the expansion,

$$
\Omega=\Omega_{0} \frac{\alpha_{2}}{4 \pi}+\Omega_{1}\left(\frac{\alpha_{2}}{4 \pi}\right)^{2}+\Omega_{2}\left(\frac{\alpha_{2}}{4 \pi}\right)^{3}+\ldots
$$

for the anomalous dimensions and beta function $\beta\left(\alpha_{2}\right)=d \alpha_{2} / d \log \mu$, we collect the coefficients necessary for resummation through NLL order in table 1. From the one-loop results given in the first column of table 1, we recover (6.3) from the ansatz in (6.4), i.e., $\hat{\Gamma}^{\prime}=\operatorname{diag}(\Gamma(0), \Gamma(2))$. 


\begin{tabular}{|c|c|c|}
\hline$\Omega$ & $\Omega_{0}$ & $\Omega_{1}$ \\
\hline$\gamma_{\text {cusp }}$ & 4 & $\left(\frac{268}{9}-\frac{4}{3} \pi^{2}\right) C_{2}(G)-\frac{80}{9} n_{G}-\frac{16}{9}$ \\
\hline$\gamma^{R}$ & $-2 C_{2}(R)$ & - \\
\hline$\gamma^{r}, \gamma^{r \prime}$ & $-\left(\frac{22}{3}-\frac{1}{6}-\frac{4}{3} n_{G}\right)$ & - \\
\hline$-\frac{\beta\left(\alpha_{2}\right)}{2 \alpha_{2}}$ & $\frac{22}{3}-\frac{1}{6}-\frac{4}{3} n_{G}$ & $\frac{259}{6}-\frac{49}{3} n_{G}-\left(\frac{3}{10}-\frac{1}{5} n_{G}\right) \frac{\alpha_{1}}{\alpha_{2}}-12 \frac{\alpha_{3}}{\alpha_{2}}+\frac{3}{2} \frac{\alpha_{t}}{\alpha_{2}}$ \\
\hline
\end{tabular}

Table 1. Expansion coefficients of $\Omega=\sum_{n=0}^{\infty}\left(\frac{\alpha_{2}}{4 \pi}\right)^{n+1} \Omega_{n}$ for the cusp and non-cusp anomalous dimensions and the $\mathrm{SU}(2)_{W}$ beta function. The appearance of $\alpha_{1}=g_{1}^{2} / 4 \pi, \alpha_{3}=g_{s}^{2} / 4 \pi$ and $\alpha_{t}=Y_{t}^{2} / 4 \pi$ in $\beta_{1}$ (and higher order in $\gamma_{\text {cusp }}, \gamma^{R}$ and $\gamma^{r}, \gamma^{r \prime}$ ) complicates the analysis beyond LL order.

\subsection{Sudakov resummation}

Let us consider the solution for coefficient scale evolution governed by (6.1). We write

$$
\vec{c}\left(\mu_{L}\right)=\hat{S}\left(\mu_{L}, \mu_{H}\right) \vec{c}\left(\mu_{H}\right)=S_{\text {cusp }}\left(\mu_{L}, \mu_{H}\right) \hat{S}_{R}\left(\mu_{L}, \mu_{H}\right) \vec{c}\left(\mu_{H}\right),
$$

where the function $S_{\text {cusp }}$ accounts for the universal scale evolution from the cusp anomalous dimension, while the matrix $\hat{S}_{R}$ accounts for scale evolution from the isospin-dependent non-cusp anomalous dimension. To LL accuracy, the solution reads

$$
S_{\text {cusp }}^{\mathrm{LL}}=\exp \left[\frac{4 \pi}{\alpha_{2}\left(\mu_{L}\right)} \frac{\gamma_{\text {cusp }, 0}}{\beta_{0}^{2}}\{r-1-r \log r\}+\frac{2 \gamma_{\text {cusp }, 0}}{\beta_{0}} \log \frac{\mu_{H}}{2 M} \log r\right], \quad S_{0}^{\mathrm{LL}}=S_{2}^{\mathrm{LL}}=1,
$$

where $r=\alpha_{2}\left(\mu_{L}\right) / \alpha_{2}\left(\mu_{H}\right)$ and $S_{0}, S_{2}$ are the diagonal elements of $\hat{S}_{R}$ in the isospin basis. In the (non-isospin) basis of operators $O_{1,2}$, we have

$$
\hat{S}=S_{\text {cusp }}\left(\begin{array}{cc}
S_{0} & \frac{2}{3}\left(S_{0}-S_{2}\right) \\
0 & S_{2}
\end{array}\right),
$$

such that mixing effects enter only at NLL order.

Let us make the explicit connection between the cusp anomalous dimension and the Sudakov double log appearing in the charged WIMP annihilation amplitude $\mathcal{M}^{+-\rightarrow \gamma \gamma}$ in (3.14). Writing $r$ as a series in $\alpha_{2}\left(\mu_{L}\right)$ we find

$$
S_{\text {cusp }}^{\mathrm{LL}}\left(m_{W}, 2 M\right)=1-\frac{\alpha_{2}}{4 \pi} 2 \gamma_{\text {cusp }, 0} \log ^{2} \frac{m_{W}}{2 M}+\left(\frac{\alpha_{2}}{4 \pi}\right)^{2} 2 \gamma_{\text {cusp }, 0}^{2} \log ^{4} \frac{m_{W}}{2 M}+\cdots+\mathcal{O}\left(\alpha_{2}^{3}\right),
$$

where in this expression $\alpha_{2}=\alpha_{2}\left(m_{W}\right)$, and the ellipsis denotes non-leading log pieces omitted above. Comparing with $\mathcal{M}^{+-\rightarrow \gamma \gamma} / 2 e^{2}$ in (3.14), we see that the Sudakov double log is exactly recovered with its coefficient tied to the cusp anomalous dimension as expected. 
The full NLL solution can be straightforwardly derived using the coefficients given in table 1. Note that beyond LL order, the running of couplings $\alpha_{1}, \alpha_{s}$ and $\alpha_{t}$ enter the RGE through $\beta_{1}$ which, however, appears only at $\mathcal{O}\left(\alpha_{2}^{3}\right)$ in $\hat{S}$ (see, e.g. Ref. [86]). The smallness of $\alpha_{2}$ thus implies that to good approximation we may investigate the numerical impact of NLL resummation with these couplings kept constant. ${ }^{9}$ The impact of LL and NLL resummation is investigated below (see figure 12).

For the present study, we focus on LL accuracy, employing the solution for $\hat{S}$ specified by (6.7) and the one-loop hard scale coefficients $c_{i}\left(\mu_{H}\right)$ given in (5.14). Our numerical investigation of corrections at LL and NLL orders indicate good perturbative convergence. The framework presented here can be readily employed for a detailed investigation of higherorder resummation relevant for WIMPs with mass in the multi-TeV range and beyond.

\section{$7 \quad$ Weak scale matching}

Having solved the RGEs in electroweak symmetric SCET, we now have the Wilson coefficients of the annihilation operators at the low scale $\mu_{L} \sim m_{W}$ in terms of those at the high scale $\mu_{H} \sim 2 M$. The final step is to match operators in this EFT, expressed in the field basis of broken electroweak symmetry, onto the quantum mechanical Hamiltonian discussed in (3.1) above. This matching will determine the elements of the RG improved $W$ matrix, which is convolved with the Sommerfeld matrix to obtain the annihilation cross section. The first task is to derive the Feynman rules for electroweak broken SCET that will then be used to compute one-loop corrections for the SCET side of the matching condition.

\subsection{Electroweak broken SCET Feynman rules}

These Feynman rules are the exact analog of what was discussed in section 4.3 except that we are now working in the electroweak broken phase. For simplicity, we again specialize to the isospin $j=1$ case, $\left(t^{a}\right)^{b c}=i \epsilon^{b a c}$. The operators are defined as in (4.19), but with gauge fields written in terms of $\gamma, Z^{0}$, and $W^{ \pm}$, introducing a dependence on $s_{W} \equiv \sin \theta_{W}$. Note that we have followed the same convention as above, defining the $c_{i}$ Wilson coefficients to be dimensionless (a 1/ $M$ factor appears in the Lagrangian (4.18)).

The Feynman rules for two gauge boson emission are

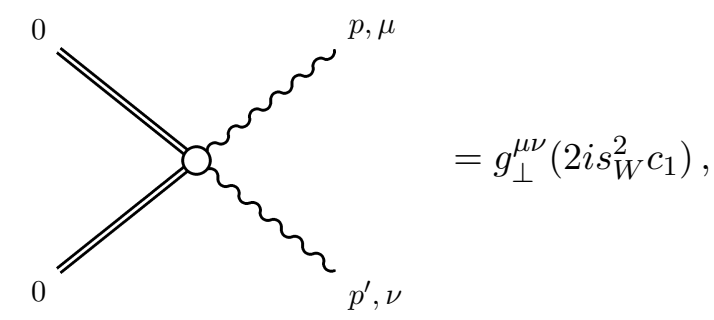

\footnotetext{
${ }^{9}$ We verify numerically that varying the fixed values of $\alpha_{1}, \alpha_{s}$ and $\alpha_{t}$ within appropriate ranges has a negligible effect on $\hat{S}$.
} 

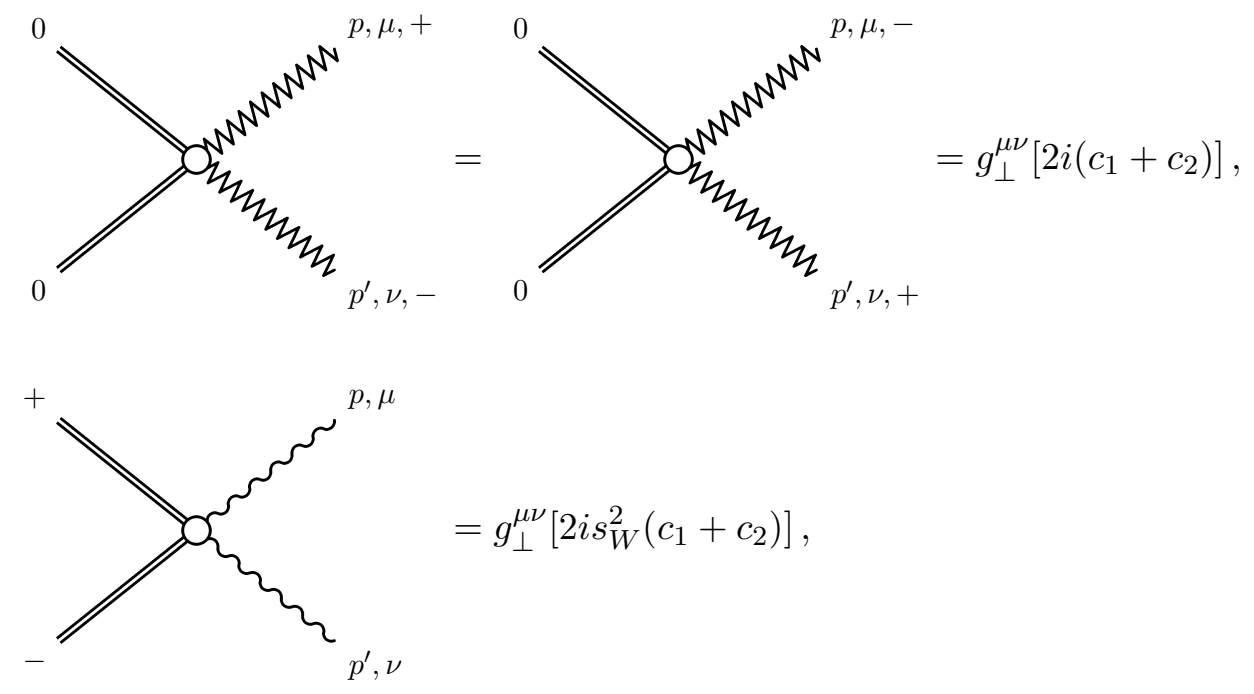

$$
=g_{\perp}^{\mu \nu}\left[2 i s_{W}^{2}\left(c_{1}+c_{2}\right)\right]
$$
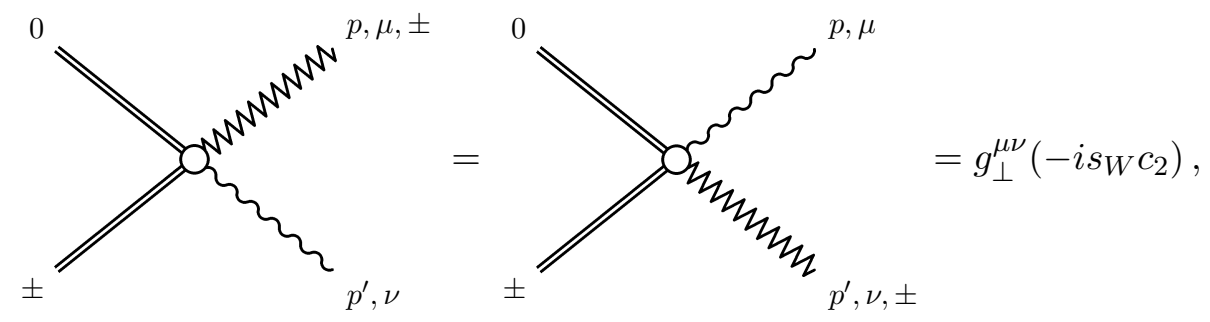

where we draw double straight lines for the heavy WIMP initial states (now being careful to distinguish the different electric charges), wavy lines for the photon, and jagged lines for the $W^{ \pm}$gauge bosons. For an additional massive hardcollinear emission from the $O_{i}$ vertex,

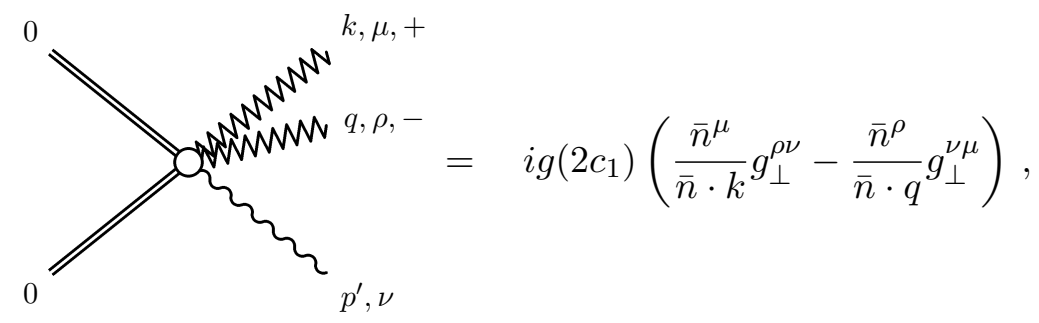

with a similar rule for two anti-hardcollinear emissions with $n \leftrightarrow \bar{n}$ as before. The interaction of soft gauge fields with heavy scalars is again given by the usual result,

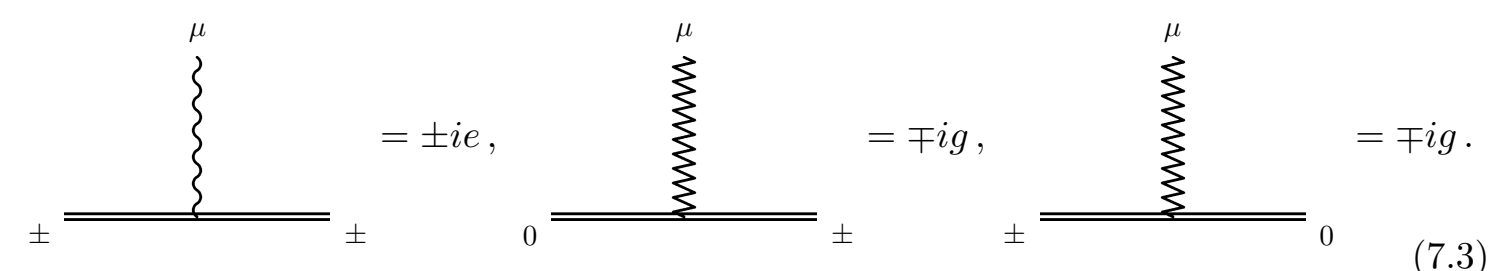

Note that rules involving the $Z^{0}$ can be inferred by changing a photon to a $Z^{0}$ and multiplying the coupling by $c_{W} / s_{W}$.

Armed with these Feynman rules, we may now compute the full one-loop matrix element for neutral and charged heavy WIMP annihilation to photons. As in section 5, 
matching must be performed between physical amplitudes, requiring onshell wavefunction factors for the external states. For the gauge field, these are the same as in the full theory, and the combination needed for this calculation $\left(Z_{1}^{W}\right)^{2}\left(Z_{2}^{W}\right)^{-2}$ is given in (3.19) above. For the heavy neutral field,

$$
Z_{\phi_{0}}=1+\frac{g^{2}}{(4 \pi)^{2}}\left[\frac{4}{\epsilon}-8 \log \frac{m_{W}}{\mu}\right]
$$

while for the heavy charged field,

$$
Z_{\phi_{ \pm}}=1+\frac{g^{2}}{(4 \pi)^{2}}\left[\frac{4}{\epsilon}-4 \log \frac{m_{W}}{\mu}-4 s_{W}^{2} \log \frac{m_{\gamma}}{\mu}-4 c_{W}^{2} \log \frac{m_{Z}}{\mu}\right] .
$$

Note that since electroweak symmetry is broken, the charged and neutral states are split due to one-loop corrections from the gauge bosons; (3.17) also applies in the EFT.

\subsection{WIMP annihilation in electroweak broken SCET}

All that remains to obtain the desired result is the computation of finite terms from matching at one loop in electroweak broken SCET. We begin by providing results for neutral WIMP annihilation. The diagrams are given in figure 8. Using the Feynman rules of the previous section we proceed to compute the one-loop matrix element for annihilation of two neutral heavy particles into two photons. Including the appropriate onshell renormalization constants, we find

$$
\begin{aligned}
& i \mathcal{M}^{00 \rightarrow \gamma \gamma}=2 i e^{2} c_{1}(\mu)+\frac{i e^{2} g^{2}}{(4 \pi)^{2}}\left\{c _ { 1 } ( \mu ) \left[C_{\text {potential }}-16 \log ^{2} \frac{m_{W}}{\mu}+32 \log \frac{2 M}{\mu} \log \frac{m_{W}}{\mu}\right.\right. \\
& \left.\left.-16 i \pi \log \frac{m_{W}}{\mu}-\frac{4 \pi^{2}}{3}\right]+c_{2}(\mu)\left[C_{\text {potential }}+16(1-i \pi) \log \frac{m_{W}}{\mu}\right]\right\} \text {, }
\end{aligned}
$$

where the only dependence on the threshold is captured by $C_{\text {potential }}$, which is given in $(3.13)$.

Let us compute the diagrams in figure 9 relevant to the charged annihilation at $k^{\mu}=$ $\delta v^{\mu}$, i.e., the threshold annihilation for charged states. The renormalized amplitude is

$$
\begin{aligned}
& i \mathcal{M}^{+-} \rightarrow \gamma \gamma \\
& =2 i e^{2}\left[c_{1}(\mu)+c_{2}(\mu)\right]+\frac{i e^{2} g^{2}}{(4 \pi)^{2}}\left\{c_{1}(\mu) \frac{8 \pi M}{m_{W}+\sqrt{-2 M \delta-i 0}}\right. \\
& \quad+\left[c_{1}(\mu)+c_{2}(\mu)\right] \frac{8 \pi s_{W}^{2} M}{m_{\gamma}}+\left[c_{1}(\mu)+c_{2}(\mu)\right] \frac{8 \pi c_{W}^{2} M}{m_{Z}} \\
& \quad+c_{1}(\mu)\left[-\frac{4 \pi^{2}}{3}+32 \log \frac{2 M}{\mu} \log \frac{m_{W}}{\mu}-16 i \pi \log \frac{m_{W}}{\mu}-16 \log ^{2} \frac{m_{W}}{\mu}\right] \\
& \left.\quad+c_{2}(\mu)\left[-\frac{4 \pi^{2}}{3}+32 \log \frac{2 M}{\mu} \log \frac{m_{W}}{\mu}-8 i \pi \log \frac{m_{W}}{\mu}-16 \log ^{2} \frac{m_{W}}{\mu}-8 \log \frac{m_{W}}{\mu}\right]\right\} .
\end{aligned}
$$

Note that we have taken $n_{G}=3$ in both (7.6) and (7.7). 


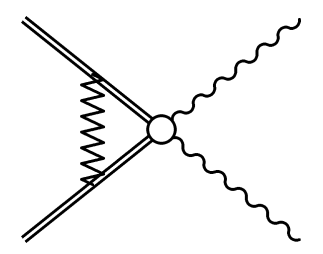

(a)

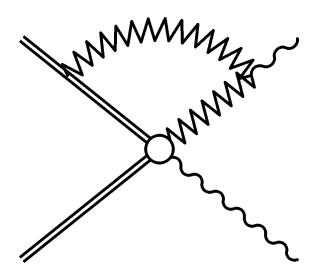

(e)

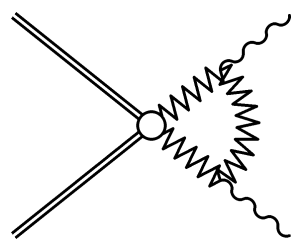

(b)

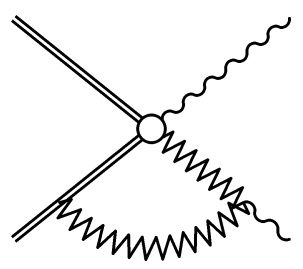

(f)

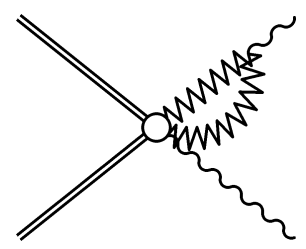

(c)

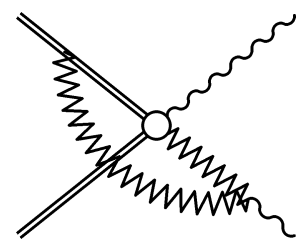

$(\mathrm{g})$

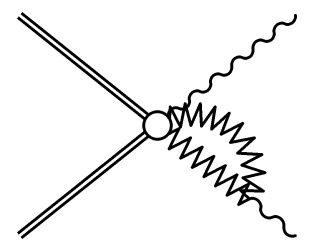

(d)

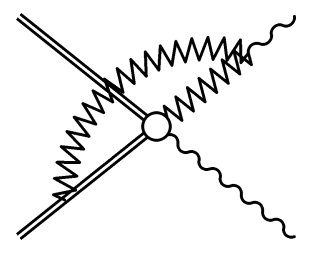

(h)

Figure 8. One-loop contributions to matrix elements of $O_{i}$ for neutral WIMPs. Double straight lines are heavy WIMPs, wavy lines are photons, and jagged lines are $W^{ \pm}$bosons.

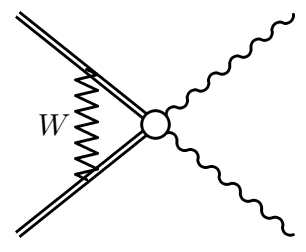

(a)

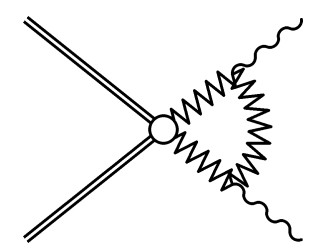

(d)

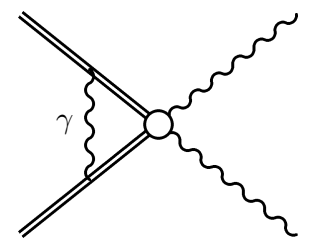

(b)

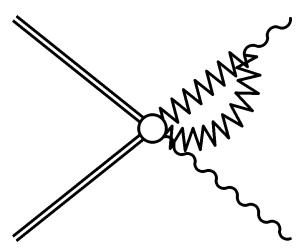

(e)

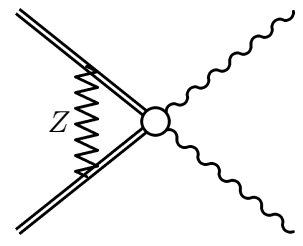

(c)

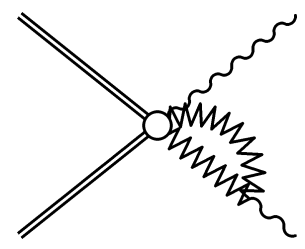

(f)

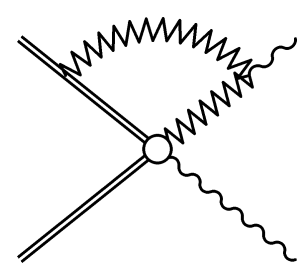

(g)

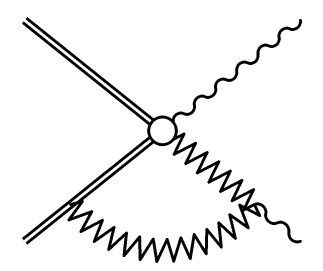

(h)

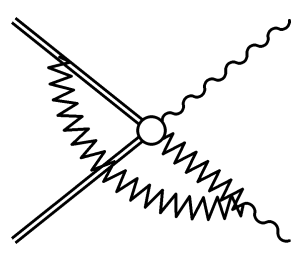

(i)

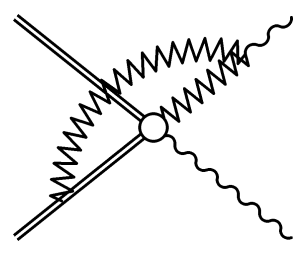

(j)

Figure 9. One-loop contributions to matrix elements of $O_{i}$, for charged WIMP annihilation. The wavy lines are photons, and the jagged lines are $W^{ \pm}$, except when explicitly labeled as a $Z^{0}$. 


\subsection{Collinear anomaly}

In evaluating the amplitudes, e.g., diagram (c) in figures 8 and 9, care must be taken to subtract a nonvanishing soft region contribution from the collinear momentum integral. This nontrivial subtraction is a remnant of nonfactorization between the collinear sectors [87], and manifests itself as residual dependence of the low-energy matrix elements (7.6), (7.7) on $\log M / \mu$, appearing at leading power in $m_{W} / M$. For problems involving a single IR scale, this residual dependence can be factorized to all orders in perturbation theory [88-93]. In the present case, we take

$$
c_{i}(\mu) \rightarrow c_{i}(\mu)\left(\frac{4 M^{2}}{\mu^{2}}\right)^{-\frac{1}{2} F\left(m_{W}, \mu\right)}
$$

where at leading order,

$$
F\left(m_{W}, \mu\right)=\frac{\alpha_{2}}{4 \pi} F_{0} \log \frac{\mu^{2}}{m_{W}^{2}}, \quad F_{0}=4 C_{2}(j) .
$$

The interplay of this so-called collinear anomaly and electroweak symmetry breaking will modify this structure beyond one loop. This order of precision is beyond phenomenological importance in the present application, and a more detailed exposition is left to future work.

\subsection{Weak scale matching results}

The quantum mechanical side of the matching computation is identical to that obtained above in section 3.4, and can be used to compute the analog of (3.27), which was derived by directly matching with the full electroweak broken theory. The absorptive parts of the potential, including the effects of resummation, are thus

$$
\begin{aligned}
w_{ \pm}=- & \frac{4 \pi \alpha^{2}}{M^{2}}\left(\frac{4 M^{2}}{\mu^{2}}\right)^{-F\left(m_{W}, \mu\right)}\left\{\left|c_{1}+c_{2}\right|^{2}+\frac{\alpha_{2}}{4 \pi}\left[| c _ { 1 } + c _ { 2 } | ^ { 2 } \left(-16 \log ^{2} \frac{m_{W}}{\mu}\right.\right.\right. \\
& \left.\left.\left.-8 \log \frac{m_{W}}{\mu}-\frac{4 \pi^{2}}{3}\right)+\operatorname{Re}\left(\left(c_{1}+c_{2}\right)^{*} c_{1}(8-8 i \pi) \log \frac{m_{W}}{\mu}\right)\right]+\mathcal{O}\left(\alpha^{2}\right)\right\} \\
w_{ \pm ; 00}=- & \frac{4 \pi \alpha^{2}}{M^{2}}\left(\frac{4 M^{2}}{\mu^{2}}\right)^{-F\left(m_{W}, \mu\right)}\left\{c_{1}\left(c_{1}+c_{2}\right)^{*}+\frac{\alpha_{2}}{4 \pi}\left[c _ { 1 } ( c _ { 1 } + c _ { 2 } ) ^ { * } \left(-16 \log ^{2} \frac{m_{W}}{\mu}\right.\right.\right. \\
& \left.-12 \log \frac{m_{W}}{\mu}+4 i \pi \log \frac{m_{W}}{\mu}-\frac{4 \pi^{2}}{3}\right)+\left|c_{1}\right|^{2}\left((4+4 i \pi) \log \frac{m_{W}}{\mu}\right) \\
& \left.\left.+\left|c_{1}+c_{2}\right|^{2}\left((8-8 i \pi) \log \frac{m_{W}}{\mu}\right)\right]+\mathcal{O}\left(\alpha^{2}\right)\right\}, \\
w_{00}=- & \frac{4 \pi \alpha^{2}}{M^{2}}\left(\frac{4 M^{2}}{\mu^{2}}\right)^{-F\left(m_{W}, \mu\right)}\left\{\left|c_{1}\right|^{2}+\frac{\alpha_{2}}{4 \pi}\left[\operatorname{Re}\left(\left(c_{1}+c_{2}\right)^{*} c_{1}(16+16 i \pi) \log \frac{m_{W}}{\mu}\right)\right.\right. \\
& \left.\left.+\left|c_{1}\right|^{2}\left(-16 \log ^{2} \frac{m_{W}}{\mu}-16 \log \frac{m_{W}}{\mu}-\frac{4 \pi^{2}}{3}\right)\right]+\mathcal{O}\left(\alpha^{2}\right)\right\},
\end{aligned}
$$

where $c_{i}(\mu)$ are the solutions (6.6) to the RG evolution equation, with high scale coefficients (5.14), and $w_{i j}$ are defined in (3.20) using plane-wave external states. These expressions accomplish a complete factorization of the scales $2 M$ and $m_{W}$, and systematically resum the large logarithms of perturbation theory. 


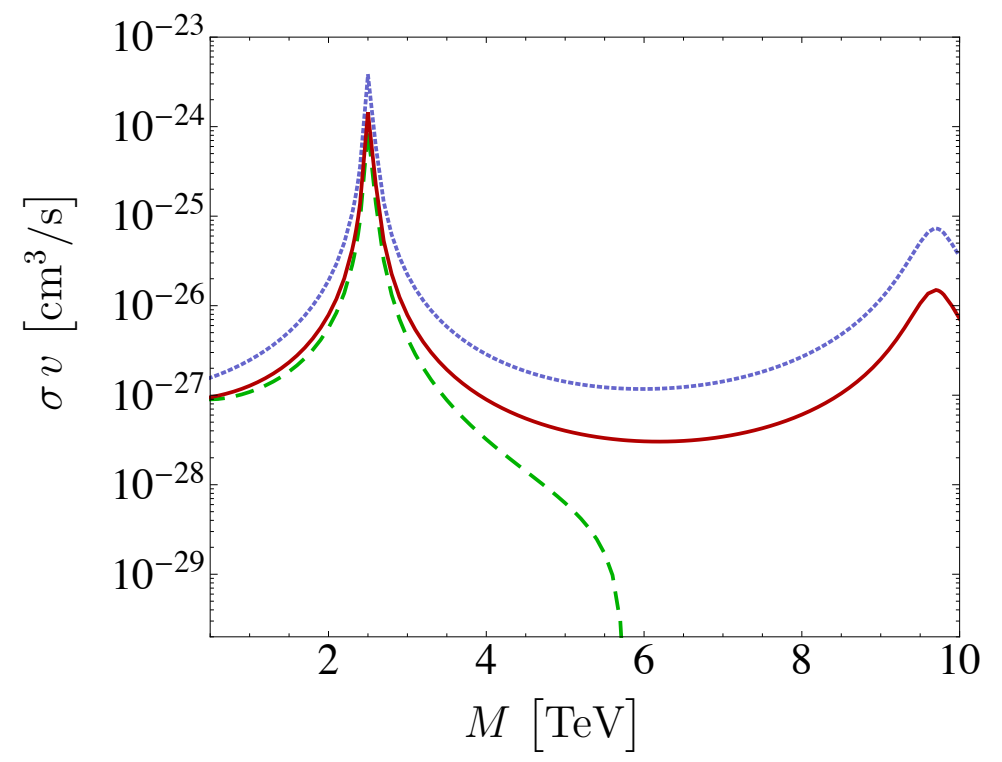

Figure 10. Sommerfeld enhanced WIMP annihilation cross sections for $\phi \phi \rightarrow \gamma \gamma$ employing three approximations. The fixed $\mathcal{O}\left(\alpha^{2}\right)$ result is shown in dotted blue. The fixed $\mathcal{O}\left(\alpha^{3}\right)$ result, including the first non-vanishing $\mathcal{O}\left(\alpha^{4}\right)$ contribution to $w_{00}$, is shown in dashed green. The LL resummed result, including one-loop matching coefficients at the hard and weak scales and resummation of the collinear anomaly contribution, is shown in solid red.

\section{Implications}

Having completed the high scale matching (5.14), RG running (6.8) and finally low scale matching (7.10), we may proceed to use the Hamiltonian to compute interesting physical observables and investigate the impact of perturbative corrections.

Figure 10 shows the Sommerfeld enhanced annihilation cross section to line photons for three approximations, taking $\delta=0.17 \mathrm{GeV}$ and $v=10^{-3}$ as above. The blue dotted and green dashed lines are fixed order results at $\mathcal{O}\left(\alpha^{2}\right)$ and $\mathcal{O}\left(\alpha^{3}\right)$, respectively, with the latter also including the first non-vanishing $\mathcal{O}\left(\alpha^{4}\right)$ contribution to $w_{00}$. The red solid line is the result including LL resummation, one-loop matching coefficients at the high and weak scales, and resummation of the collinear anomaly contribution. The uncertainty from scale variation would not be resolved on this log plot, hence we only show the central value and discuss perturbative uncertainties below. As previously discussed the fixed $\mathcal{O}\left(\alpha^{3}\right)$ result (green dashed) becomes negative for $M \gtrsim 6 \mathrm{TeV}$, indicating a breakdown in perturbation theory.

There is a robust suppression of the resummed result due to the LL correction from the (universal) cusp anomalous dimension. We give the ratios of the Sommerfeld enhanced fixed order cross sections to the resummed cross section, $(\sigma v)_{\text {tree }} /(\sigma v)_{\mathrm{LL}}$ and $(\sigma v)_{1 \text {-loop }} /(\sigma v)_{\mathrm{LL}}$, in figure 11. At $M=3 \mathrm{TeV}$ the resummed result is suppressed by a factor of $\sim 3$ with respect to tree level.

To illustrate the impact of higher order perturbative corrections, let us investigate the residual renormalization scale dependence of the absorptive part of the potential at LL and 


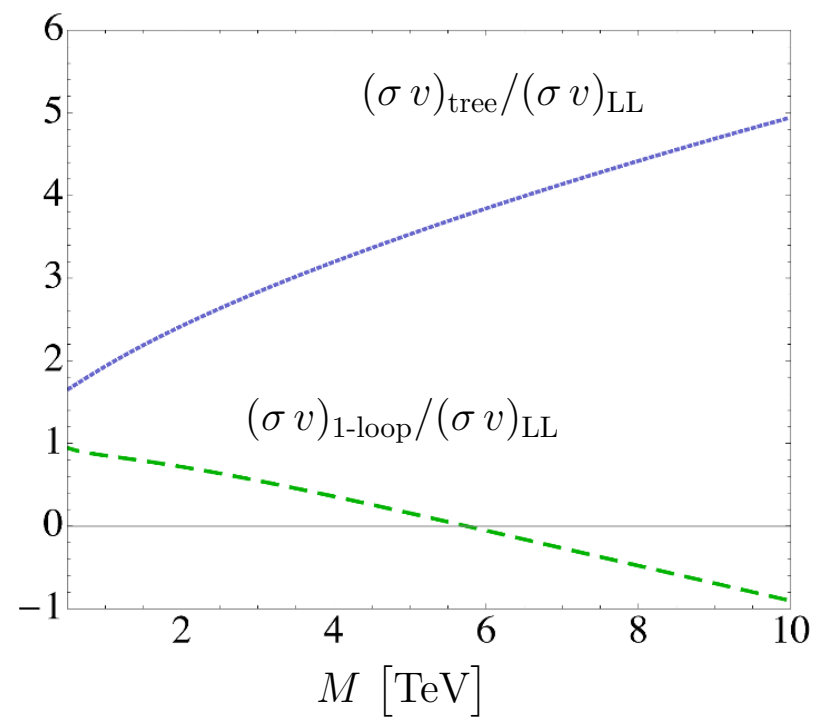

Figure 11. This plot shows the ratio of $(\sigma v)_{\text {tree }} /(\sigma v)_{\mathrm{LL}}$ (blue dotted) and $(\sigma v)_{1-\operatorname{loop}} /(\sigma v)_{\mathrm{LL}}$ (green dashed) including the effects of the Sommerfeld enhancement. The LL resummed result here includes one-loop matching coefficients at the hard and weak scales and resummation of the collinear anomaly contribution.

NLL accuracy. We focus here on $w_{ \pm}$which has the largest impact on the neutral WIMP annihilation cross section to photons. For LL order, we include the LL solution to the RG evolution and tree-level matching coefficients at the hard and intermediate scales, but neglect the collinear anomaly contribution. For NLL order, we include the NLL solution to the RG evolution, tree-level matching coefficients at the hard and intermediate scales and full resummation of the collinear anomaly contribution.

The results of this study are shown in figure 12 where we plot $w_{ \pm}$in units of $M^{2} / \pi \alpha^{2}$ so that the tree-level result is unity. The purple and grey bands are the LL and NLL results, respectively, where the uncertainty is from the combined variation of scales $m_{W} / 2<\mu_{L}<$ $2 m_{W}$ and $M<\mu_{H}<4 M$. For comparison, we also include the fixed $\mathcal{O}\left(\alpha^{3}\right)$ result (dashed green line), and the resummed result (red band) employed for $\sigma v$ in figures 11 and 11 above. The fixed order result has no explicit $\mu$ dependence, while the uncertainty for the red band is from the combined variation of scales $m_{W} / 2<\mu_{L}<2 m_{W}$ and $M<\mu_{H}<4 M$. The sizable uncertainty in the LL result (purple band) is due to the scale variation of the Sudakov double log, which cancels at NLL order with the variation of the $\mathcal{O}(\alpha)$ contribution from the collinear anomaly. Note that the NLL result (gray band) and the resummed result employed in figures 10 and 11 are degenerate." as the last sentence of that paragraph.

The resummed results capture the large $\alpha \log ^{2} \frac{2 M}{m_{W}}$ contribution through scale evolution of the hard matching coefficients $c_{i}(\mu)$, which enter quadratically in (7.10). The fixed order result, on the other hand, has the large $\alpha \log ^{2} \frac{2 M}{m_{W}}$ contribution but appearing only linearly in $w_{ \pm}$. For $M \gtrsim 7 \mathrm{TeV}$ the missing contributions result in $w_{ \pm}$becoming positive $\left(-M^{2} w_{ \pm} / \pi \alpha^{2}\right.$ becoming negative) which translates to a negative $\sigma v$ in figure 11 above. The resummation of large logarithms is necessary to control perturbative corrections for WIMP masses in the $\mathrm{TeV}$ regime. 


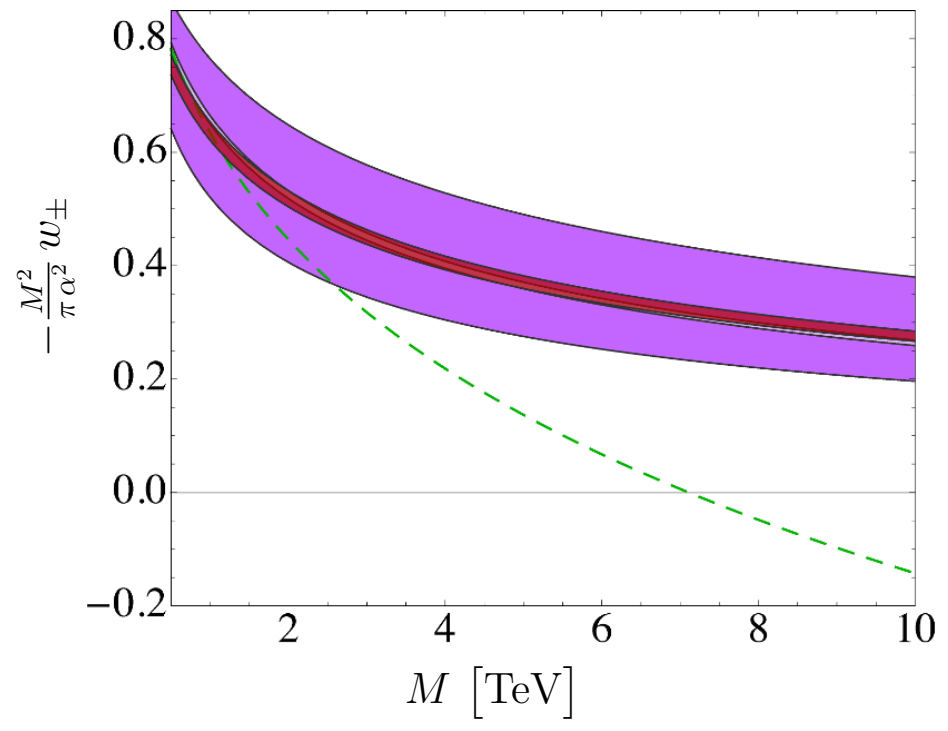

Figure 12. The LL (purple) and NLL (gray) results for $-M^{2} w_{ \pm} / \pi \alpha^{2}$ with estimated error bands combined from varying $m_{W} / 2<\mu_{L}<2 m_{W}$ and $M<\mu_{H}<4 M$. For comparison, we also include the fixed $\mathcal{O}\left(\alpha^{3}\right)$ result (dashed green line), and the resummed result (red band) employed for $\sigma v$ in figures 10 and 11 above.

\section{Summary}

We have constructed a general EFT framework to analyze heavy WIMP annihilation. The factorization accomplished in (7.10) provides a systematically improvable framework in which to compute annihilation observables. By separating the WIMP, $M$, and electroweak, $m_{W}$, scales, the EFT allows hard scale matching conditions to be efficiently computed in the electroweak symmetric theory, while low-scale matching conditions and long-distance wavefunction analysis may be performed in simpler effective theories.

At the same time, large logarithms that would otherwise lead to a breakdown in perturbation theory are systematically resummed by solving the RGEs derived from the effective theory operators in the intermediate, soft collinear, effective theory. In particular, a universal suppression of heavy WIMP annihilations is traced to the cusp anomalous dimension governing effective theory operators. We provided details of the operator construction, hard scale matching, and renormalization of this effective theory.

Below the electroweak scale, we mapped the problem to the relevant quantum mechanical Hamiltonian describing the nonrelativistic WIMP system. The relevant matching conditions in this effective theory were computed, and used to derive expressions for the absorptive part of the potential representing the chosen annihilation channel. This two-step matching procedure recovers the results of a one-step matching procedure at fixed order in perturbation theory, but systematically resums large logarithms. Having fully determined the low energy theory in a controlled perturbative expansion, we computed an illustrative observable represented by the low-velocity annihilation rate to two photon final states.

The EFT framework presented here can be applied to a broad class of models and signatures. Details of the particular UV completion are encoded in the hard scale matching 
coefficients, while heavy WIMP spin symmetry implies the existence of universal features that are associated with the remaining physical scales. In particular, the dominant effect from including the loop corrections derives from a universal factor that is independent of the spin and electroweak quantum numbers of the WIMP. Disentangling the different energy scales in a sequence of effective theories allows the separate treatment of physical effects associated with the hard annihilation process, the Sudakov suppression, and the Sommerfeld enhancement of annihilation observables. Subleading perturbative, power and velocity corrections may be systematically incorporated.

As a concrete application, we focused attention on a heavy scalar $\mathrm{SU}(2)_{W}$ triplet annihilating into photons. While fixed order perturbation theory breaks down in the $\mathrm{TeV}$ WIMP mass regime, our resummed results exhibit a convergent perturbative expansion. The leading effect relative to tree level is represented by a universal Sudakov suppression, which at $M=3 \mathrm{TeV}$ implies a resummed cross section that is reduced by a factor $\sim 3$.

In a forthcoming paper [71], we will examine observational consequences in more detail, including the computation for triplet fermion annihilation and reinterpretation of constraints on this process using theoretically reliable cross sections. This work demonstrates that accounting for large logarithms through resummation is necessary for robust predictions of the heavy WIMP annihilation cross section - this is of clear importance in order to compare theory and indirect detection experiments.

Note added. While this work was in the final stages of preparation, [94] appeared which provides some partial results on resummation neglecting the effects of electroweak symmetry breaking for heavy WIMP dark matter. We also became aware of another work on a similar topic [95], which is to appear soon.

\section{Acknowledgments}

We thank Thomas Becher, Matthew Dolan, Matthias Neubert, Michael Peskin, Jon Walsh, Hua-Xing Zhu for insightful discussions. In particular, we are grateful to Mariangela Lisanti, Aaron Pierce, and Tracy Slatyer for permission to use the Sommerfeld enhancement code developed for [41]. MB is supported by the Alexander von Humboldt Foundation. MB thanks SLAC, where some of this research was performed, for support and hospitality. TC is supported by DoE contract number DE-AC02-76SF00515 and by an LHC Theory Initiative Postdoctoral Fellowship, under the National Science Foundation grant PHY-0969510. TC thanks the KITP in Santa Barbara where some of this research was performed and for the support from the National Science Foundation under Grant No. NSF PHY11-25915. TC also thanks the MITP in Mainz where additional work was performed. RH is supported by the DOE Grant No. DE-FG02-13ER41958. TC and RH thank the Aspen Center for Physics where this work was initiated for the support under NSF Grant No. NSF PHY10-66293. MS acknowledges support from a Bloomenthal Fellowship at the University of Chicago and from the Office of Science, Office of High Energy Physics, of the U.S. Department of Energy under contract DE-AC02-05CH11231. 
Open Access. This article is distributed under the terms of the Creative Commons Attribution License (CC-BY 4.0), which permits any use, distribution and reproduction in any medium, provided the original author(s) and source are credited.

\section{References}

[1] J.L. Feng et al., Planning the future of U.S. particle physics (Snowmass 2013): chapter 4: cosmic frontier, arXiv:1401.6085 [INSPIRE].

[2] T. Moroi and L. Randall, Wino cold dark matter from anomaly mediated SUSY breaking, Nucl. Phys. B 570 (2000) 455 [hep-ph/9906527] [INSPIRE].

[3] G.B. Gelmini and P. Gondolo, Neutralino with the right cold dark matter abundance in (almost) any supersymmetric model, Phys. Rev. D 74 (2006) 023510 [hep-ph/0602230] [INSPIRE].

[4] B.S. Acharya, G. Kane, S. Watson and P. Kumar, A non-thermal WIMP miracle, Phys. Rev. D 80 (2009) 083529 [arXiv:0908.2430] [InSPIRE].

[5] T. Moroi, M. Nagai and M. Takimoto, Non-thermal production of wino dark matter via the decay of long-lived particles, JHEP 07 (2013) 066 [arXiv:1303.0948] [INSPIRE].

[6] R. Easther, R. Galvez, O. Ozsoy and S. Watson, Supersymmetry, nonthermal dark matter and precision cosmology, Phys. Rev. D 89 (2014) 023522 [arXiv:1307.2453] [INSPIRE].

[7] G. Bertone, D. Hooper and J. Silk, Particle dark matter: evidence, candidates and constraints, Phys. Rept. 405 (2005) 279 [hep-ph/0404175] [INSPIRE].

[8] T. Cohen, J. Kearney, A. Pierce and D. Tucker-Smith, Singlet-doublet dark matter, Phys. Rev. D 85 (2012) 075003 [arXiv:1109.2604] [InSPIRE].

[9] C. Cheung, L.J. Hall, D. Pinner and J.T. Ruderman, Prospects and blind spots for neutralino dark matter, JHEP 05 (2013) 100 [arXiv:1211.4873] [INSPIRE].

[10] N. Arkani-Hamed, A. Delgado and G.F. Giudice, The well-tempered neutralino, Nucl. Phys. B 741 (2006) 108 [hep-ph/0601041] [INSPIRE].

[11] M. Cirelli, N. Fornengo and A. Strumia, Minimal dark matter, Nucl. Phys. B 753 (2006) 178 [hep-ph/0512090] [INSPIRE].

[12] M. Cirelli, A. Strumia and M. Tamburini, Cosmology and astrophysics of minimal dark matter, Nucl. Phys. B 787 (2007) 152 [arXiv:0706.4071] [inSPIRE].

[13] M. Cirelli, R. Franceschini and A. Strumia, Minimal dark matter predictions for galactic positrons, anti-protons, photons, Nucl. Phys. B 800 (2008) 204 [arXiv:0802.3378] [INSPIRE].

[14] M. Cirelli and A. Strumia, Minimal dark matter: model and results, New J. Phys. 11 (2009) 105005 [arXiv: 0903.3381] [INSPIRE].

[15] J. Hisano, S. Matsumoto, M. Nagai, O. Saito and M. Senami, Non-perturbative effect on thermal relic abundance of dark matter, Phys. Lett. B 646 (2007) 34 [hep-ph/0610249] [INSPIRE].

[16] A. Hryczuk, R. Iengo and P. Ullio, Relic densities including Sommerfeld enhancements in the MSSM, JHEP 03 (2011) 069 [arXiv:1010.2172] [INSPIRE]. 
[17] T. Hur, D.-W. Jung, P. Ko and J.Y. Lee, Electroweak symmetry breaking and cold dark matter from strongly interacting hidden sector, Phys. Lett. B 696 (2011) 262 [arXiv:0709.1218] [INSPIRE].

[18] C. Kilic, T. Okui and R. Sundrum, Vectorlike confinement at the LHC, JHEP 02 (2010) 018 [arXiv:0906.0577] [INSPIRE].

[19] M.T. Frandsen and F. Sannino, iTIMP: isotriplet Technicolor Interacting Massive Particle as dark matter, Phys. Rev. D 81 (2010) 097704 [arXiv:0911.1570] [InSPIRE].

[20] Y. Bai and R.J. Hill, Weakly interacting stable pions, Phys. Rev. D 82 (2010) 111701 [arXiv: 1005.0008] [INSPIRE].

[21] N. Craig, The state of supersymmetry after run I of the LHC, arXiv:1309.0528 [INSPIRE].

[22] CMS collaboration, Search for dark matter and large extra dimensions in pp collisions yielding a photon and missing transverse energy, Phys. Rev. Lett. 108 (2012) 261803 [arXiv:1204.0821] [INSPIRE].

[23] CMS collaboration, Search for dark matter and large extra dimensions in monojet events in pp collisions at $\sqrt{s}=7 \mathrm{TeV}$, JHEP 09 (2012) 094 [arXiv:1206.5663] [INSPIRE].

[24] ATLAS collaboration, Search for dark matter in events with a hadronically decaying $W$ or $Z$ boson and missing transverse momentum in pp collisions at $\sqrt{s}=8 \mathrm{TeV}$ with the ATLAS detector, Phys. Rev. Lett. 112 (2014) 041802 [arXiv: 1309.4017] [INSPIRE].

[25] ATLAS collaboration, Search for charginos nearly mass degenerate with the lightest neutralino based on a disappearing-track signature in pp collisions at $\sqrt{s}=8 \mathrm{TeV}$ with the ATLAS detector, Phys. Rev. D 88 (2013) 112006 [arXiv:1310.3675] [INSPIRE].

[26] ATLAS collaboration, Search for dark matter in events with a $Z$ boson and missing transverse momentum in pp collisions at $\sqrt{s}=8 \mathrm{TeV}$ with the ATLAS detector, Phys. Rev. D 90 (2014) 012004 [arXiv: 1404.0051] [INSPIRE].

[27] CMS collaboration, Searches for electroweak neutralino and chargino production in channels with Higgs, $Z$ and $W$ bosons in pp collisions at 8 TeV, Phys. Rev. D 90 (2014) 092007 [arXiv: 1409.3168] [INSPIRE].

[28] M. Low and L.-T. Wang, Neutralino dark matter at 14 TeV and $100 \mathrm{TeV}$, JHEP 08 (2014) 161 [arXiv:1404.0682] [INSPIRE].

[29] M. Cirelli, F. Sala and M. Taoso, Wino-like minimal dark matter and future colliders, JHEP 10 (2014) 033 [arXiv: 1407.7058] [INSPIRE].

[30] J. Hisano, K. Ishiwata, N. Nagata and T. Takesako, Direct detection of electroweak-interacting dark matter, JHEP 07 (2011) 005 [arXiv: 1104.0228] [INSPIRE].

[31] R.J. Hill and M.P. Solon, Universal behavior in the scattering of heavy, weakly interacting dark matter on nuclear targets, Phys. Lett. B 707 (2012) 539 [arXiv:1111.0016] [INSPIRE].

[32] R.J. Hill and M.P. Solon, WIMP-nucleon scattering with heavy WIMP effective theory, Phys. Rev. Lett. 112 (2014) 211602 [arXiv: 1309.4092] [INSPIRE].

[33] R.J. Hill and M.P. Solon, Standard model anatomy of WIMP dark matter direct detection I: weak-scale matching, arXiv:1401.3339 [INSPIRE].

[34] J. Billard, L. Strigari and E. Figueroa-Feliciano, Implication of neutrino backgrounds on the reach of next generation dark matter direct detection experiments, Phys. Rev. D 89 (2014) 023524 [arXiv: 1307 .5458] [INSPIRE]. 
[35] J. Hisano, S. Matsumoto and M.M. Nojiri, Unitarity and higher order corrections in neutralino dark matter annihilation into two photons, Phys. Rev. D 67 (2003) 075014 [hep-ph/0212022] [INSPIRE].

[36] J. Hisano, S. Matsumoto and M.M. Nojiri, Explosive dark matter annihilation, Phys. Rev. Lett. 92 (2004) 031303 [hep-ph/0307216] [INSPIRE].

[37] J. Hisano, S. Matsumoto, M.M. Nojiri and O. Saito, Non-perturbative effect on dark matter annihilation and $\gamma$ ray signature from galactic center, Phys. Rev. D 71 (2005) 063528 [hep-ph/0412403] [INSPIRE].

[38] P. Ciafaloni et al., Weak corrections are relevant for dark matter indirect detection, JCAP 03 (2011) 019 [arXiv: 1009.0224] [InSPIRE].

[39] A. Hryczuk and R. Iengo, The one-loop and sommerfeld electroweak corrections to the Wino dark matter annihilation, JHEP 01 (2012) 163 [Erratum ibid. 1206 (2012) 137] [arXiv:1111.2916] [INSPIRE].

[40] HESS collaboration, A. Abramowski et al., Search for photon line-like signatures from dark matter annihilations with H.E.S.S., Phys. Rev. Lett. 110 (2013) 041301 [arXiv:1301.1173] [INSPIRE].

[41] T. Cohen, M. Lisanti, A. Pierce and T.R. Slatyer, Wino dark matter under siege, JCAP 10 (2013) 061 [arXiv:1307.4082] [INSPIRE].

[42] J. Fan and M. Reece, In Wino veritas? Indirect searches shed light on neutralino dark matter, JHEP 10 (2013) 124 [arXiv:1307.4400] [INSPIRE].

[43] A. Hryczuk, I. Cholis, R. Iengo, M. Tavakoli and P. Ullio, Indirect detection analysis: Wino dark matter case study, JCAP 07 (2014) 031 [arXiv:1401.6212] [INSPIRE].

[44] T. Becher, R.J. Hill, B.O. Lange and M. Neubert, External operators and anomalous dimensions in soft collinear effective theory, Phys. Rev. D 69 (2004) 034013 [hep-ph/0309227] [INSPIRE].

[45] T. Becher and M. Neubert, On the structure of infrared singularities of gauge-theory amplitudes, JHEP 06 (2009) 081 [Erratum ibid. 1311 (2013) 024] [arXiv:0903.1126] [INSPIRE].

[46] T. Becher and M. Neubert, Infrared singularities of QCD amplitudes with massive partons, Phys. Rev. D 79 (2009) 125004 [Erratum ibid. D 80 (2009) 109901] [arXiv:0904.1021] [INSPIRE].

[47] M. Beneke, P. Falgari and C. Schwinn, Soft radiation in heavy-particle pair production: all-order colour structure and two-loop anomalous dimension, Nucl. Phys. B 828 (2010) 69 [arXiv: 0907.1443] [INSPIRE].

[48] W.E. Caswell and G.P. Lepage, Effective lagrangians for bound state problems in QED, QCD and other field theories, Phys. Lett. B 167 (1986) 437 [InSPIRE].

[49] E. Eichten and B.R. Hill, An effective field theory for the calculation of matrix elements involving heavy quarks, Phys. Lett. B 234 (1990) 511 [INSPIRE].

[50] M. Neubert, Heavy quark symmetry, Phys. Rept. 245 (1994) 259 [hep-ph/9306320] [INSPIRE].

[51] A.V. Manohar and M.B. Wise, Heavy quark physics, Cambridge University Press, Cambridge U.K. (2000). 
[52] C.W. Bauer, S. Fleming and M.E. Luke, Summing Sudakov logarithms in $B \rightarrow X_{s} \gamma$ in effective field theory, Phys. Rev. D 63 (2000) 014006 [hep-ph/0005275] [INSPIRE].

[53] C.W. Bauer, S. Fleming, D. Pirjol and I.W. Stewart, An effective field theory for collinear and soft gluons: heavy to light decays, Phys. Rev. D 63 (2001) 114020 [hep-ph/0011336] [INSPIRE].

[54] C.W. Bauer and I.W. Stewart, Invariant operators in collinear effective theory, Phys. Lett. B 516 (2001) 134 [hep-ph/0107001] [INSPIRE].

[55] C.W. Bauer, D. Pirjol and I.W. Stewart, Soft collinear factorization in effective field theory, Phys. Rev. D 65 (2002) 054022 [hep-ph/0109045] [INSPIRE].

[56] C.W. Bauer, D. Pirjol and I.W. Stewart, Power counting in the soft collinear effective theory, Phys. Rev. D 66 (2002) 054005 [hep-ph/0205289] [INSPIRE].

[57] R.J. Hill and M. Neubert, Spectator interactions in soft collinear effective theory, Nucl. Phys. B 657 (2003) 229 [hep-ph/0211018] [INSPIRE].

[58] J. Chay and C. Kim, Collinear effective theory at subleading order and its application to heavy-light currents, Phys. Rev. D 65 (2002) 114016 [hep-ph/0201197] [INSPIRE].

[59] M. Beneke, A.P. Chapovsky, M. Diehl and T. Feldmann, Soft collinear effective theory and heavy to light currents beyond leading power, Nucl. Phys. B 643 (2002) 431 [hep-ph/0206152] [INSPIRE].

[60] L. Randall and R. Sundrum, Out of this world supersymmetry breaking, Nucl. Phys. B 557 (1999) 79 [hep-th/9810155] [INSPIRE].

[61] G.F. Giudice, M.A. Luty, H. Murayama and R. Rattazzi, Gaugino mass without singlets, JHEP 12 (1998) 027 [hep-ph/9810442] [INSPIRE].

[62] J.D. Wells, PeV-scale supersymmetry, Phys. Rev. D 71 (2005) 015013 [hep-ph/0411041] [INSPIRE].

[63] N. Arkani-Hamed and S. Dimopoulos, Supersymmetric unification without low energy supersymmetry and signatures for fine-tuning at the LHC, JHEP 06 (2005) 073 [hep-th/0405159] [INSPIRE].

[64] G.F. Giudice and A. Romanino, Split supersymmetry, Nucl. Phys. B 699 (2004) 65 [Erratum ibid. B 706 (2005) 65-89] [hep-ph/0406088] [INSPIRE].

[65] A. Pierce, Dark matter in the finely tuned minimal supersymmetric standard model, Phys. Rev. D 70 (2004) 075006 [hep-ph/0406144] [INSPIRE].

[66] A. Arvanitaki, N. Craig, S. Dimopoulos and G. Villadoro, Mini-split, JHEP 02 (2013) 126 [arXiv: 1210.0555] [INSPIRE].

[67] N. Arkani-Hamed, A. Gupta, D.E. Kaplan, N. Weiner and T. Zorawski, Simply unnatural supersymmetry, arXiv:1212.6971 [INSPIRE].

[68] L.J. Hall, Y. Nomura and S. Shirai, Spread supersymmetry with Wino LSP: gluino and dark matter signals, JHEP 01 (2013) 036 [arXiv:1210.2395] [INSPIRE].

[69] G. Kane, P. Kumar, R. Lu and B. Zheng, Higgs mass prediction for realistic string/M theory vacua, Phys. Rev. D 85 (2012) 075026 [arXiv:1112.1059] [InSPIRE].

[70] M. Ibe and T.T. Yanagida, The lightest Higgs boson mass in pure gravity mediation model, Phys. Lett. B 709 (2012) 374 [arXiv:1112.2462] [INSPIRE]. 
[71] M. Bauer, T. Cohen, R.J. Hill and M.P. Solon, Soft-collinear effective theory for wino dark matter annihilation, to appear.

[72] R.J. Hill and G.P. Lepage, Order $\left(\alpha^{2} \Gamma\right)$ binding effects in orthopositronium decay, Phys. Rev. D 62 (2000) 111301 [hep-ph/0003277] [INSPIRE].

[73] M. Beneke, C. Hellmann and P. Ruiz-Femenia, Non-relativistic pair annihilation of nearly mass degenerate neutralinos and charginos I. General framework and S-wave annihilation, JHEP 03 (2013) 148 [Erratum ibid. 1310 (2013) 224] [arXiv:1210.7928] [INSPIRE].

[74] C. Hellmann and P. Ruiz-Femenía, Non-relativistic pair annihilation of nearly mass degenerate neutralinos and charginos II. P-wave and next-to-next-to-leading order S-wave coefficients, JHEP 08 (2013) 084 [arXiv:1303.0200] [INSPIRE].

[75] N. Arkani-Hamed, D.P. Finkbeiner, T.R. Slatyer and N. Weiner, A theory of dark matter, Phys. Rev. D 79 (2009) 015014 [arXiv:0810.0713] [INSPIRE].

[76] Particle Data Group collaboration, K. Olive et al., Review of particle physics, Chin. Phys. C 38 (2014) 090001 [inSPIRE].

[77] W.F.L. Hollik, Radiative corrections in the standard model and their role for precision tests of the electroweak theory, Fortsch. Phys. 38 (1990) 165 [INSPIRE].

[78] J.-y. Chiu, F. Golf, R. Kelley and A.V. Manohar, Electroweak Sudakov corrections using effective field theory, Phys. Rev. Lett. 100 (2008) 021802 [arXiv:0709.2377] [INSPIRE].

[79] J.-y. Chiu, F. Golf, R. Kelley and A.V. Manohar, Electroweak corrections in high energy processes using effective field theory, Phys. Rev. D 77 (2008) 053004 [arXiv:0712.0396] [INSPIRE].

[80] J.-y. Chiu, R. Kelley and A.V. Manohar, Electroweak corrections using effective field theory: applications to the LHC, Phys. Rev. D 78 (2008) 073006 [arXiv:0806.1240] [INSPIRE].

[81] A. Manohar, B. Shotwell, C. Bauer and S. Turczyk, Non-cancellation of electroweak logarithms in high-energy scattering, Phys. Lett. B 740 (2015) 179 [arXiv:1409.1918] [INSPIRE].

[82] V.A. Smirnov, Applied asymptotic expansions in momenta and masses, Springer Tacts in Modern Physics volume 177, Springer, Germany (2002).

[83] A.V. Manohar, T. Mehen, D. Pirjol and I.W. Stewart, Reparameterization invariance for collinear operators, Phys. Lett. B 539 (2002) 59 [hep-ph/0204229] [INSPIRE].

[84] R.J. Hill, G. Lee, G. Paz and M.P. Solon, NRQED Lagrangian at order $1 / M^{4}$, Phys. Rev. D 87 (2013) 053017 [arXiv: 1212.4508] [INSPIRE].

[85] D.M. Pierce, J.A. Bagger, K.T. Matchev and R.-j. Zhang, Precision corrections in the minimal supersymmetric standard model, Nucl. Phys. B 491 (1997) 3 [hep-ph/9606211] [INSPIRE].

[86] L.G. Almeida et al., Comparing and counting logs in direct and effective methods of QCD resummation, JHEP 04 (2014) 174 [arXiv:1401.4460] [INSPIRE].

[87] T. Becher, R.J. Hill and M. Neubert, Soft collinear messengers: A New mode in soft collinear effective theory, Phys. Rev. D 69 (2004) 054017 [hep-ph/0308122] [INSPIRE].

[88] T. Becher and M. Neubert, Drell-Yan production at small $q_{T}$, transverse parton distributions and the collinear anomaly, Eur. Phys. J. C 71 (2011) 1665 [arXiv:1007.4005] [InSPIRE]. 
[89] T. Becher, M. Neubert and D. Wilhelm, Electroweak gauge-boson production at small $q_{T}$ : infrared safety from the collinear anomaly, JHEP 02 (2012) 124 [arXiv:1109.6027] [INSPIRE].

[90] J.-y. Chiu, A. Jain, D. Neill and I.Z. Rothstein, The rapidity renormalization group, Phys. Rev. Lett. 108 (2012) 151601 [arXiv:1104.0881] [INSPIRE].

[91] T. Becher, G. Bell and M. Neubert, Factorization and resummation for jet broadening, Phys. Lett. B 704 (2011) 276 [arXiv:1104.4108] [INSPIRE].

[92] J.-Y. Chiu, A. Jain, D. Neill and I.Z. Rothstein, A formalism for the systematic treatment of rapidity logarithms in quantum field theory, JHEP 05 (2012) 084 [arXiv:1202.0814] [INSPIRE].

[93] T. Becher and M. Neubert, Factorization and NNLL resummation for Higgs production with a jet veto, JHEP 07 (2012) 108 [arXiv:1205.3806] [INSPIRE].

[94] M. Baumgart, I.Z. Rothstein and V. Vaidya, On the annihilation rate of WIMPs, arXiv: 1409.4415 [INSPIRE].

[95] G. Ovanesyan, T.R. Slatyer and I.W. Stewart, Heavy dark matter annihilation from effective field theory, arXiv:1409.8294 [INSPIRE]. 